\title{
On quasi-linear equation problems involving critical and singular nonlinearities
}

\section{Yanbin Sang*, Xiaorong Luo}

Department of Mathematics, School of Science, North University of China, Taiyuan, Shanxi 030051, China.

\section{Abstract}

We consider the singular boundary value problem

$$
\left\{\begin{array}{l}
-\operatorname{div}\left(|x|^{-a p}|\nabla u|^{p-2} \nabla u\right)=h(x) \frac{u^{-\gamma}}{|x|^{b(1-\gamma)}}+\mu \frac{u^{p^{*}-1}}{|x|^{b p^{*}}} \text { in } \Omega \backslash\{0\}, \\
u>0 \quad \text { in } \Omega \backslash\{0\}, \\
u=0 \quad \text { on } \partial \Omega,
\end{array}\right.
$$

where $\Omega \subset \mathbb{R}^{N}(N \geqslant 3)$ is a bounded domain such that $0 \in \Omega, 0<\gamma<1,0 \leqslant a<\frac{N-p}{p}, a \leqslant b<a+1, p^{*}:=p^{*}(a, b)=$ $\frac{N p}{N-(1+a-b) p}$, and $h(x)$ is a given function. Based on different assumptions, using variational methods and Ekeland's principle, we admit that this problem possesses two positive solutions. (C)2017 All rights reserved.

Keywords: Critical exponent, p-Laplacian operator, extremal value, singular nonlinearity. 2010 MSC: 5A15, 35B33, 35J62.

\section{Introduction}

In this paper, we are concerned with the following problem:

$$
\left\{\begin{array}{l}
-\operatorname{div}\left(|x|^{-a p}|\nabla u|^{p-2} \nabla u\right)=h(x) \frac{u^{-\gamma}}{|x|^{(1-\gamma)}}+\mu \frac{\mathfrak{u}^{p^{*}-1}}{|x|^{p^{*}}} \text { in } \Omega \backslash\{0\}, \\
u>0 \quad \text { in } \Omega \backslash\{0\}, \\
u=0 \quad \text { on } \partial \Omega,
\end{array}\right.
$$

where $\Omega$ is a bounded domain in $\mathbb{R}^{N}(N \geqslant 3)$ with smooth boundary $\partial \Omega$ such that $0 \in \Omega$. $\mu>0$ is a parameter, $0<\gamma<1,0 \leqslant a<\frac{N-p}{p}, 1<p<N, a \leqslant b<a+1$, and $p^{*}:=p^{*}(a, b)=\frac{N p}{N-(1+a-b) p}$ is the critical Hardy-Sobolev exponent. Throughout our paper, we assume that $h \in C(\bar{\Omega})$ and $h(x)>0$.

\footnotetext{
*Corresponding author

Email addresses: syb6662004@163.com (Yanbin Sang), 993237546@qq.com (Xiaorong Luo)
} 
Let $W_{a}^{1, p}(\Omega)$ be the completion of $C_{0}^{\infty}(\Omega)$ with respect to the norm $\|u\|_{a}$, where

$$
\|\mathrm{u}\|_{\mathrm{a}}=\int_{\Omega}\left(|x|^{-\mathrm{ap}}|\nabla \mathrm{u}|^{\mathrm{p}} \mathrm{dx}\right)^{\frac{1}{\mathrm{p}}} .
$$

In recent years, considerable attention has been attracted to quasilinear elliptic problems [1, 2, 8$10,12-17,25]$. In [13], Ghoussoub and Yuan studied the existence of positive solutions for the following quasi-linear equation:

$$
\left\{\begin{array}{l}
-\Delta_{\mathfrak{p}} u=\lambda|\mathfrak{u}|^{r-2} \mathfrak{u}+\mu \frac{|\mathfrak{u}|^{q-2}}{|x|^{s}} \mathfrak{u}, \quad x \in \Omega, \\
\mathfrak{u}(x)=0, \quad x \in \partial \Omega,
\end{array}\right.
$$

where $1<p<N, 0 \leqslant s \leqslant p, p \leqslant q \leqslant \frac{N-s}{N-p}$ and $p \leqslant r \leqslant \frac{N p}{N-p}$.

We would like to mention the results of $[11,18,26]$, which motivated us to discuss (1.1). In [11], Deng and Huang considered the following quasilinear elliptic equation

$$
-\operatorname{div}\left(|x|^{-a p}|\nabla u|^{p-2} \nabla u\right)=\frac{\mu+h(x)}{|x|^{(a+1) p}}|u|^{p-2} u+k(x) \frac{|u|^{p^{*}-2} u}{|x|^{b p^{*}}}, \quad x \in \mathbb{R}^{N},
$$

where $1<p<N, 0 \leqslant a<\frac{N-p}{p}, a \leqslant b<a+1,0 \leqslant \mu<\left(\frac{N-p}{p}-a\right)^{p}, p^{*}:=p^{*}(a, b)=\frac{N p}{N-(1+a-b) p}$, and $k$ and $h$ are continuously bounded functions. Under some assumptions on $h$ and $k$, several multiplicity theorems of (1.2) were established. Furthermore, Kang [18] studied the following quasilinear problem

$$
\left\{\begin{array}{l}
-\operatorname{div}\left(|x|^{-a p}|\nabla u|^{p-2} \nabla u\right)-\mu \frac{|u|^{p-2} u}{|x|^{p(a+1)}}=\frac{|u|^{*}(a, b)-2 u}{|x|^{p^{*}(a, b)}}+\lambda \frac{|u|^{q-2} u}{|x|^{\mathbf{d}^{*}(a, d)}} \text { in } \Omega \backslash\{0\}, \\
u=0 \quad \text { on } \partial \Omega
\end{array}\right.
$$

where $\Omega \subset \mathbb{R}^{N}$ is a bounded domain with smooth boundary, $0 \in \Omega, N \geqslant 3, \lambda>0,1<p<N$, $0 \leqslant \mu<\left(\frac{N-p}{p}-a\right)^{p}, 0 \leqslant a<\frac{N-p}{p}, a \leqslant b, d<a+1, p \leqslant q<p^{*}(a, d)=\frac{N p}{N-p(a+1-d)}$. He investigated the extremal functions and gave some estimates. We should point out that Xuan [26] has provided some properties of eigenvalues of the following quasilinear problem

$$
\left\{\begin{array}{l}
-\operatorname{div}\left(|x|^{-a p}|\nabla u|^{p-2} \nabla u\right)=\lambda|x|^{-(a+1) p+c}|u|^{p-2} \mathfrak{u} \text { in } \Omega, \\
u=0 \quad \text { on } \partial \Omega
\end{array}\right.
$$

where $\Omega \subset \mathbb{R}^{N}$ is an open bounded domain with $C^{1}$ boundary, $0 \in \Omega, 1<p<N, 0 \leqslant a<\frac{N-p}{p}$, and $c>0$.

On the other hand, Giacomoni et al. [14] established the multiplicity result of (1.1) when $h(x) \equiv \lambda$, $\mathrm{a}=\mathrm{b}=0$, and $\mu \equiv 1$ by critical point theory and a lower-upper solution method. Moreover, Loc and Schmitt [19] studied the following singular problem:

$$
\left\{\begin{array}{l}
-\Delta_{p} u=a(x) g(u)+\lambda h(u), \quad x \in \Omega, \\
u(x)=0, \quad x \in \partial \Omega, \\
u(x)>0, \quad x \in \Omega,
\end{array}\right.
$$

where $p>1, g(u)$ is a singular term, $a \in L^{\infty}(\Omega), \lambda$ is a parameter and $h(u)$ is a continuous function. They constructed lower-upper solutions to show the problem (1.3) has one weak solution in $W_{0}^{1, p}(\Omega)$. We note that when $p=2$, the multiplicity of positive solutions for problem (1.1) has been considered by Sun and $\mathrm{Wu}[22]$, Sun and $\mathrm{Li}[21]$, and Chen and Chen [6, 7].

In this paper, we will establish some existence and multiplicity theorems for (1.1) when $\mu \in\left(0, \mu^{*}\right)$ for some $\mu^{*}>0$ and give the lower bounds for $\mu^{*}=\mu^{*}\left(\Omega, \gamma, p^{*}, h(x)\right)>0$. 


\section{Preliminaries}

Throughout this paper, define $\|\mathrm{u}\|_{s}^{s}=\int_{\Omega}\left(|x|^{-b}|\mathfrak{u}|\right)^{s} \mathrm{~d} x$. We denote by the first eigenfunction $e_{1}$ with $-\operatorname{div}\left(|x|^{-a p}|\nabla u|^{p-2} \nabla u\right)=\lambda_{1} \frac{e_{1}^{p-1}}{|x|^{b p}}$ in $\Omega, e_{1} \mid \partial \Omega=0,0 \leqslant e_{1} \leqslant 1$.

We set

$$
S_{a}=\inf \left\{\frac{\int_{\Omega}|x|^{-a p}|\nabla u|^{p} d x}{\left(\int_{\Omega}|x|^{-b p^{*}}|u|^{p^{*}} d x\right)^{\frac{p}{p^{*}}}} \mid u \in W_{a}^{1, p}(\Omega), u \neq 0\right\} .
$$

The infimum can be achieved by the function $U^{*}(x)$, where $U^{*}$ is the radially symmetric ground state of the following limiting problem

$$
\left\{\begin{array}{l}
-\operatorname{div}\left(|x|^{-a p}|\nabla u|^{p-2} \nabla u\right)=\frac{u^{p^{*}-1}}{|x|^{b p^{*}}} \text { in } \mathbb{R}^{N} \backslash\{0\}, \\
u(x)>0 \text { in } \mathbb{R}^{N} \backslash\{0\}, \\
u \rightarrow 0 \text { as }|x| \longrightarrow \infty
\end{array}\right.
$$

The functional associated to (1.1) is

$$
\mathrm{I}_{\mu}(\mathrm{u})=\frac{1}{\mathrm{p}} \int_{\Omega}|x|^{-\mathrm{ap}}|\nabla u|^{p}-\frac{1}{1-\gamma} \int_{\Omega} \mathrm{h}(\mathrm{x})\left(|x|^{-\mathrm{b}}|\mathrm{u}|\right)^{1-\gamma}-\frac{\mu}{\mathrm{p}^{*}} \int_{\Omega} \frac{|\mathrm{u}|^{p^{*}}}{|x|^{b p^{*}}}, \quad \forall u \in W_{\mathrm{a}}^{1, p}(\Omega) .
$$

We introduce the constraint set

$$
\mathcal{N}_{\mu}=\left\{t(u) u: u \in W_{a}^{1, p}(\Omega) \backslash\{0\}\right\},
$$

where $t(u)$ are the zeros of the following map

$$
\begin{aligned}
\mathrm{t} \longrightarrow \varphi(\mathrm{t}, \mathrm{u}) & =\frac{1}{\mathrm{t}^{*}-1} \frac{\mathrm{d}}{\mathrm{dt}} \mathrm{I}_{\mu}(\mathrm{tu}) \\
& =\mathrm{t}^{\mathrm{p}-\mathrm{p}^{*}} \int_{\Omega}|x|^{-\mathrm{ap}}|\nabla u|^{p}-\mathrm{t}^{-\gamma-\mathrm{p}^{*}+1} \int_{\Omega} \mathrm{h}(\mathrm{x})\left(|x|^{-\mathrm{b}}|\mathrm{u}|\right)^{1-\gamma}-\mu \int_{\Omega} \frac{|\mathrm{u}|^{\mathrm{p}^{*}}}{|x|^{b p^{*}}} .
\end{aligned}
$$

In order to obtain our results, split $\mathcal{N}_{\mu}$ into the following three parts

$$
\begin{aligned}
& \mathcal{N}_{\mu}^{+}=\left\{v=t(u) u \in \mathcal{N}_{\mu}:\left(p-p^{*}\right)\|v\|_{a}^{p}+\left(p^{*}+\gamma-1\right) \int_{\Omega} h(x)\left(|x|^{-b}|v|\right)^{1-\gamma} d x>0\right\}, \\
& \mathcal{N}_{\mu}^{0}=\left\{v=t(u) u \in \mathcal{N}_{\mu}:\left(p-p^{*}\right)\|v\|_{a}^{p}+\left(p^{*}+\gamma-1\right) \int_{\Omega} h(x)\left(|x|^{-b}|v|\right)^{1-\gamma} d x=0\right\}, \\
& \mathcal{N}_{\mu}^{-}=\left\{v=t(u) u \in \mathcal{N}_{\mu}:\left(p-p^{*}\right)\|v\|_{a}^{p}+\left(p^{*}+\gamma-1\right) \int_{\Omega} h(x)\left(|x|^{-b}|v|\right)^{1-\gamma} d x<0\right\} .
\end{aligned}
$$

A function $u$ is called a solution of (1.1) if $u \in W_{a}^{1, p}(\Omega)$ such that $u(x)>0$ a.e. in $\Omega$ and

$$
\int_{\Omega}|x|^{-a \mathfrak{p}}|\nabla u|^{p-2} \nabla u \nabla \phi-\int_{\Omega} \frac{h(x)\left(|x|^{-b}\right)^{1-\gamma}}{u^{\gamma}} \phi-\mu \int_{\Omega} \frac{u^{p^{*}-1}}{|x|^{b p^{*}}} \phi=0, \quad \forall \phi \in W_{a}^{1, p}(\Omega) .
$$

Lemma 2.1. Assume that $\mu \in(0, \mathrm{~T})$, where

$$
\mathrm{T}=\left(\frac{\gamma-1+\mathrm{p}}{\gamma+\mathrm{p}^{*}-1}\right)\left(\frac{\mathrm{p}^{*}-\mathrm{p}}{\gamma+\mathrm{p}^{*}-1}\right)^{\frac{\mathrm{p}^{*}-\mathrm{p}}{\mathrm{p}-1+\gamma}}\left(\frac{1}{\|\mathrm{~h}\|_{\infty}}\right)^{\frac{\mathrm{p}^{*}-\mathrm{p}}{\mathrm{p}-1+\gamma}}\left(\frac{\mathrm{S}_{\lambda}}{|\Omega| \frac{\mathrm{p}(1+\mathrm{a}-\mathrm{b})}{\mathrm{N}}}\right)^{\frac{\gamma+\mathrm{p}^{*}-1}{\mathrm{p}-1+\gamma}},
$$

then $\mathcal{N}_{\mu}^{0}=\{0\}$. Furthermore, for every $u \in W_{a}^{1, p}(\Omega) \backslash\{0\}, \varphi(t, u)$ has exactly two zeros $\mathrm{t}^{\mp}(\mathrm{u})$ such that

$$
0<t^{-}(u)<t^{+}(u), t^{-}(u) u \in \mathcal{N}_{\mu}^{+}, t^{+}(u) u \in \mathcal{N}_{\mu}^{-} \text {. }
$$


Proof.

(1) Define $\varphi:(0, \infty) \times\left\{W_{\mathrm{a}}^{1, \mathrm{p}}(\Omega) \backslash\{0\}\right\} \longrightarrow \mathbb{R}$ by $(2.1)$. Let $\varphi^{\prime}(\mathrm{t}, \mathrm{u})=0$, then

$$
t=\left[\frac{\left(p^{*}-p\right)\|u\|_{a}^{p}}{\left(\gamma+p^{*}-1\right) \int_{\Omega} h(x)\left(|x|^{-b}|u|\right)^{1-\gamma} d x}\right]^{-\frac{1}{p-1+\gamma}}:=t_{\max , u} .
$$

We can deduce that $\varphi^{\prime}(t, u)>0$ when $0<t<t_{\max , u}$ and $\varphi^{\prime}(t, u)<0$ when $t>t_{\max , u}$. Furthermore, we have

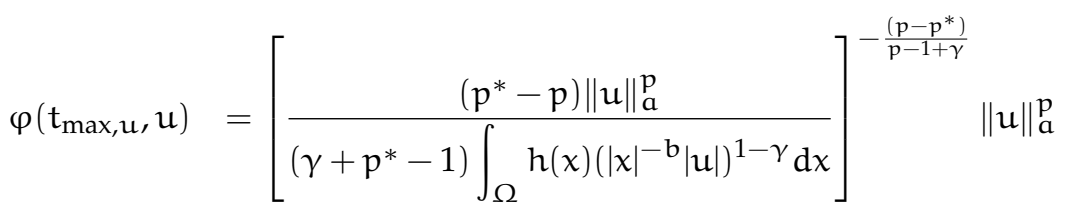

$$
\begin{aligned}
& -\left[\frac{\left(p^{*}-p\right)\|u\|_{a}^{p}}{\left(\gamma+p^{*}-1\right) \int_{\Omega} h(x)\left(|x|^{-b}|u|\right)^{1-\gamma} d x}\right]^{-\frac{\left(-\gamma-p^{*}+1\right)}{p-1+\gamma}} \\
& \times \int_{\Omega} h(x)\left(|x|^{-b}|u|\right)^{1-\gamma} d x-\mu \int_{\Omega}\left(|x|^{-b}|u|\right)^{p^{*}} d x
\end{aligned}
$$

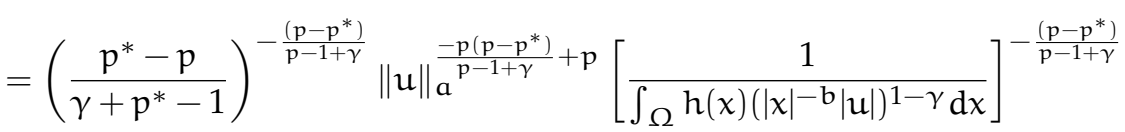

$$
\begin{aligned}
& -\left(\frac{p^{*}-p}{\gamma+p^{*}-1}\right)^{\frac{\gamma+p^{*}-1}{p-1+\gamma}}\|u\|_{a}^{\frac{p}{p}\left(\gamma+p^{*}-1\right)}\left[\frac{1}{p^{-1+\gamma}}\left[\frac{1}{\int_{\Omega} h(x)\left(|x|^{-b}|u|\right)^{1-\gamma} d x}\right]^{\frac{\gamma+p^{*}-1}{p-1+\gamma}}\right. \\
& \times \int_{\Omega} h(x)\left(|x|^{-b}|u|\right)^{1-\gamma} \mathrm{dx} \\
& -\mu \int_{\Omega}\left(|x|^{-b}|u|\right)^{p^{*}} \mathrm{dx} \\
& =\left(\frac{\gamma-1+p}{\gamma+p^{*}-1}\right)\left(\frac{p^{*}-p}{\gamma+p^{*}-1}\right)^{\frac{p^{*}-p}{p-1+\gamma}} \frac{\|u\|_{a}^{\frac{p\left(p^{*}-1+\gamma\right)}{p-1+\gamma}}}{\left(\int_{\Omega} h(x)\left(|x|^{-b}|u|\right)^{1-\gamma} d x\right)^{\frac{p^{*}-p}{p+\gamma-1}}}-\mu \int_{\Omega}\left(\left.|x|^{-b}|u|\right|^{p^{*}} d x .\right.
\end{aligned}
$$

Since $\|u\|_{\mathfrak{a}}^{p} \geqslant S_{a}\|u\|_{p^{*}}^{p}$ for every $u \in W_{a}^{1, p}(\Omega) \backslash\{0\}$, in view of Hölder inequality, we have

$$
\begin{aligned}
\int_{\Omega} h(x)\left(|x|^{-b}|u|\right)^{1-\gamma} \mathrm{d} x & \leqslant\left(\int_{\Omega}\left(|x|^{-\mathbf{b}}|\mathrm{u}|\right)^{(1-\gamma) \frac{\mathrm{p}^{*}}{1-\gamma}} \mathrm{dx}\right)^{\frac{1-\gamma}{\mathrm{p}^{*}}}\left(\int_{\Omega} \mathrm{h}(\mathrm{x})^{\frac{\mathrm{p}^{*}}{\mathrm{p}^{*}-1+\gamma}} \mathrm{dx}\right)^{\frac{\mathrm{p}^{*}-1+\gamma}{\mathrm{p}^{*}}} \\
& \leqslant\|\mathrm{u}\|_{\mathrm{p}^{*}}^{1-\gamma}\|\mathrm{h}\|_{\infty}|\Omega|^{\frac{\mathrm{p}^{*}-1+\gamma}{\mathrm{p}^{*}}} \\
& \leqslant\left(\frac{\|\mathrm{u}\|_{\mathrm{a}}}{\sqrt[p]{\mathrm{S}_{\mathrm{a}}}}\right)^{1-\gamma}\|\mathrm{h}\|_{\infty}|\Omega|^{\frac{\mathrm{p}^{*}-1+\gamma}{\mathrm{p}^{*}}}
\end{aligned}
$$

Therefore,

$$
\begin{aligned}
& \varphi\left(t_{\max , u}, \mathfrak{u}\right) \geqslant\left(\frac{\gamma-1+p}{\gamma+p^{*}-1}\right)\left(\frac{p^{*}-p}{\gamma+p^{*}-1}\right)^{\frac{p^{*}-p}{p-1+\gamma}} \frac{\|u\|_{a}^{\frac{p\left(p^{*}-1+\gamma\right)}{p^{-1+\gamma}}}}{\left[\left(\frac{\|u\|_{a}}{\sqrt[p]{S_{a}}}\right)^{1-\gamma}\|h\|_{\infty}|\Omega|^{\frac{p^{*}-1+\gamma}{p^{*}}}\right]^{\frac{p^{*}-p}{p-1+\gamma}}}-\mu\left(\frac{\|u\|_{a}}{\sqrt[p]{S_{a}}}\right)^{p^{*}} \\
& =\left[\left(\frac{\gamma-1+p}{\gamma+p^{*}-1}\right)\left(\frac{p^{*}-p}{\gamma+p^{*}-1}\right)^{\frac{p^{*}-p}{p-1+\gamma}}\left(\frac{1}{\|h\|_{\infty}}\right)^{\frac{p^{*}-p}{p-1+\gamma}} \frac{\left(\sqrt[p]{S_{a}}\right)^{\frac{(1-\gamma)\left(p^{*}-p\right)}{p-1+\gamma}}}{|\Omega|^{\frac{\left(p^{*}-1+\gamma\right)\left(p^{*}-p\right)}{p^{*}(p-1+\gamma)}}}-\frac{\mu}{\left(\sqrt[p]{S_{a}}\right)^{p^{*}}}\right]\|u\|_{a}^{p^{*}}
\end{aligned}
$$




$$
:=E(\mu)\|u\|_{a}^{p^{*}} .
$$

We can see that

$$
\begin{aligned}
E(\mu)=0 \Leftrightarrow & \mu=\left(\frac{\gamma-1+p}{\gamma+p^{*}-1}\right)\left(\frac{p^{*}-p}{\gamma+p^{*}-1}\right)^{\frac{p^{*}-p}{p-1+\gamma}}\left(\frac{1}{\|h\|_{\infty}}\right)^{\frac{p^{*}-p}{p-1+\gamma}} \frac{\left(\sqrt[p]{S_{a}}\right)^{\frac{(1-\gamma)\left(p^{*}-p\right)}{p-1+\gamma}+p^{*}}}{|\Omega|^{\frac{\left(p^{*}-1+\gamma\right)\left(\mathfrak{p}^{*}-p\right)}{p^{*}(\mathfrak{p}-1+\gamma)}}} \\
= & \left(\frac{\gamma-1+p}{\gamma+p^{*}-1}\right)\left(\frac{p^{*}-p}{\gamma+p^{*}-1}\right)^{\frac{p^{*}-p}{p-1+\gamma}}\left(\frac{1}{\|h\|_{\infty}}\right)^{\frac{p^{*}-p}{p-1+\gamma}}\left(\frac{S_{a}}{|\Omega|^{\frac{(1+a-b) p}{N}}}\right)^{\frac{\gamma+p^{*}-1}{p-1+\gamma}} \\
& :=\mathrm{T} .
\end{aligned}
$$

So for $\mu \in(0, T)$, we have that $E(\mu)>0$. Thus, $\varphi(t, u)$ has exactly two zeros $0<t^{-}(u)<t_{\max , u}<t^{+}(u)$ such that $\varphi^{\prime}\left(t^{-}(u), u\right)>0>\varphi^{\prime}\left(t^{+}(u), u\right)$. Since $\varphi\left(t^{-}(u), u\right)=0, \varphi^{\prime}\left(t^{-}(u), u\right)>0$. Then $t^{-}(u) u \in \mathcal{N}_{\mu}$ and

$$
\left(p-p^{*}\right)\left[t^{-}(u)\right]^{p-p^{*}-1}\|u\|_{a}^{p}+\left(\gamma+p^{*}-1\right)\left[t^{-}(u)\right]^{-\gamma-p^{*}} \int_{\Omega} h(x)\left(|x|^{-b}|u|\right)^{1-\gamma} d x>0 .
$$

By (2.1), we obtain that

$$
\left(p-p^{*}\right)\left\|t^{-}(u) u\right\|_{a}^{p}+\left(\gamma+p^{*}-1\right) \int_{\Omega} h(x)\left(\left|t^{-}(u) u \| x\right|^{-b}\right)^{1-\gamma} d x>0 .
$$

That is $\mathrm{t}^{-}(\mathrm{u}) \mathrm{u} \in \mathcal{N}_{\mathfrak{u}}^{+}$. Similarly, we get that $\mathrm{t}^{+}(\mathrm{u}) \mathrm{u} \in \mathcal{N}_{\mu}^{-}$.

(2) Now, we will prove that $\mathcal{N}_{\mu}^{0}=\{0\}$. Assume by contradiction that there exists $u_{*} \in W_{a}^{1, p}(\Omega) \backslash\{0\}$ such that $\mathrm{t}\left(\mathrm{u}_{*}\right) \mathrm{u}_{*} \in \mathcal{N}_{\mu}^{0}, \mathrm{t}\left(\mathrm{u}_{*}\right) \mathrm{u}_{*} \neq 0$. Then

$$
\left[t\left(u_{*}\right)\right]^{p-p^{*}}\left\|u_{*}\right\|_{\mathfrak{a}}^{p}-\left[t\left(u_{*}\right)\right]^{-\gamma-p^{*}+1} \int_{\Omega} h(x)\left(|x|^{-b}\left|u_{*}\right|\right)^{1-\gamma} d x-\mu \int_{\Omega}\left|u_{*}\right|^{p^{*}} d x=0,
$$

and

$$
\left(p-p^{*}\right)\left\|t\left(u_{*}\right) u_{*}\right\|_{a}^{p}+\left(\gamma+p^{*}-1\right) \int_{\Omega} h(x)\left(|x|^{-b}\left|t\left(u_{*}\right) u_{*}\right|\right)^{1-\gamma} d x=0 .
$$

Hence, for $\mu \in(0, T)$ and $u_{*} \in W_{a}^{1, p}(\Omega) \backslash\{0\}$, it follows from (2.2) and (2.3) that

$$
\begin{aligned}
0< & E(\mu)\left\|u_{*}\right\|_{a}^{p^{*}} \\
\leqslant & \left(\frac{\gamma-1+p}{\gamma+p^{*}-1}\right)\left(\frac{p^{*}-p}{\gamma+p^{*}-1}\right)^{\frac{p^{*}-p}{p-1+\gamma}} \frac{\left\|u_{*}\right\|_{a}^{\frac{p\left(p^{*}-1+\gamma\right)}{p-1+\gamma}}}{\left(\int_{\Omega} h(x)\left(|x|^{-b}\left|\mathfrak{u}_{*}\right|\right)^{1-\gamma} d x\right)^{\frac{p^{*}-p}{p-1+\gamma}}}-\mu \int_{\Omega} \frac{\left|u_{*}\right|^{p^{*}}}{|x|^{b p^{*}}} d x \\
= & \left(\frac{\gamma-1+p}{\gamma+p^{*}-1}\right)\left(\frac{p^{*}-p}{\gamma+p^{*}-1}\right)^{\frac{p^{*}-p}{p-1+\gamma}} \frac{\left\|u_{*}\right\|_{a}^{\frac{p\left(p^{*}-1+\gamma\right)}{p-1+\gamma}}}{\left(\frac{-p+p^{*}}{\gamma+p^{*}-1}\left[t\left(u_{*}\right)\right]^{p-1+\gamma}\left\|u_{*}\right\|_{a}^{p}\right)^{\frac{p^{*}-p}{p-1+\gamma}}} \\
& -\left(\frac{p-1+\gamma}{\gamma+p^{*}-1}\right)\left[t\left(u_{*}\right)\right]^{p-p^{*}}\left\|u_{*}\right\|_{a}^{p}=0,
\end{aligned}
$$

this is a contradiction. Therefore, $t\left(u_{*}\right) u_{*}=0$.

Lemma 2.2. Assume that $\mu \in(0, \mathrm{~T})$, then $\mathcal{N}_{\mu}$ has the following properties

$$
\|V\|_{a}>A(\mu)>A_{0}>\|v\|_{a}, \forall V \in \mathcal{N}_{\mu}^{-}, v \in \mathcal{N}_{\mu}^{+},
$$


where

$$
\begin{gathered}
A_{0}=\left[\left(\frac{\gamma+p^{*}-1}{p^{*}-p}\right)\left(\frac{1}{\sqrt[p]{S_{a}}}\right)^{1-\gamma}\|h\|_{\infty}|\Omega|^{\frac{p^{*}-1+\gamma}{p^{*}}}\right]^{\frac{1}{p-1+\gamma}}, \\
A(\mu)=\left[\frac{(p+\gamma-1)\left(\sqrt[p]{S_{a}}\right)^{p^{*}}}{\mu\left(\gamma+p^{*}-1\right)}\right]^{\frac{1}{p^{*}-p}} .
\end{gathered}
$$

Proof. If $v \in \mathcal{N}_{\mu}^{+}$, then

$$
\left(p^{*}-p\right)\|v\|_{a}^{p}<\left.\left(\gamma+p^{*}-1\right) \int_{\Omega} h(x)\left(|x|^{-b} \mid v\right)\right|^{1-\gamma} d x .
$$

From (2.2), we deduce that

$$
\begin{aligned}
\|v\|_{a}^{p} & <\frac{\gamma+p^{*}-1}{p^{*}-p} \int_{\Omega} h(x)\left(|x|^{-b}|v|\right)^{1-\gamma} d x \\
& \leqslant\left(\frac{\gamma+p^{*}-1}{p^{*}-p}\right)\left(\frac{\|v\|_{a}}{\sqrt[p]{S_{a}}}\right)^{1-\gamma}\|h\|_{\infty}|\Omega|^{\frac{p^{*}-1+\gamma}{p^{*}}}
\end{aligned}
$$

which yields

$$
\begin{aligned}
\|v\|_{a} & <\left[\left(\frac{\gamma+p^{*}-1}{p^{*}-p}\right)\left(\frac{1}{\sqrt[p]{S_{a}}}\right)^{1-\gamma}\|h\|_{\infty}|\Omega|^{\frac{p^{*}-1+\gamma}{p^{*}}}\right]^{\frac{1}{p-1+\gamma}} \\
& :=A_{0}
\end{aligned}
$$

If $V \in \mathcal{N}_{\mu}^{-}$, we have

$$
\begin{aligned}
\|\mathrm{V}\|_{\mathrm{a}}^{\mathrm{p}} & <\left(\frac{\gamma+\mathrm{p}^{*}-1}{\mathrm{p}+\gamma-1}\right) \mu \int_{\Omega}\left(|x|^{-\mathrm{b}}|\mathrm{V}|\right)^{p^{*}} \mathrm{~d} x \\
& \leqslant\left(\frac{\gamma+\mathrm{p}^{*}-1}{\mathrm{p}+\gamma-1}\right) \mu\left(\frac{\|\mathrm{V}\|_{\mathrm{a}}}{\sqrt[p]{S_{\mathrm{a}}}}\right)^{\mathrm{p}^{*}},
\end{aligned}
$$

which implies

$$
\|V\|_{a}>\left[\frac{(p+\gamma-1)\left(\sqrt[p]{S_{a}}\right)^{p^{*}}}{\mu\left(\gamma+p^{*}-1\right)}\right]^{\frac{1}{p^{*}-p}}:=A(\mu) .
$$

In the following, we show that

$$
\mu=\mathrm{T} \Leftrightarrow \mathrm{A}(\mu)=\mathrm{A}_{0} .
$$

In fact

$$
\begin{aligned}
& \mu=T \Leftrightarrow A(\mu)=\left(\frac{\gamma+p^{*}-1}{\gamma-1+p}\right)^{\frac{1}{p^{*}-p}}\left(\frac{\gamma+p^{*}-1}{p^{*}-p}\right)^{\frac{1}{p-1+\gamma}}\left(\|h\|_{\infty}\right)^{\frac{1}{p-1+\gamma}} \\
& \times \frac{|\Omega|^{\frac{p(1+a-b)}{N}} \frac{\left(\gamma+p^{*}-1\right)}{(p-1+\gamma)\left(p^{*}-p\right)}}{\left(S_{a}\right)^{\frac{\gamma+p^{*}-1}{(p-1+\gamma)\left(p^{*}-p\right)}}}\left(\frac{p+\gamma-1}{\gamma+p^{*}-1}\right)^{\frac{1}{p^{*}-p}}\left(\sqrt[p]{S_{a}}\right)^{\frac{p^{*}}{p^{*}-p}}
\end{aligned}
$$

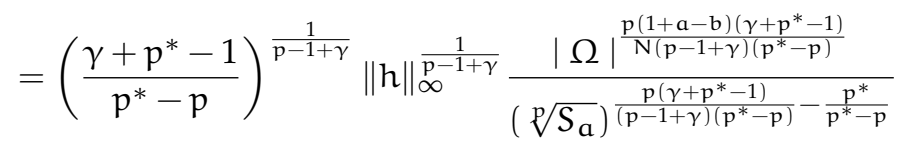

$$
\begin{aligned}
& =\left[\left(\frac{\gamma+p^{*}-1}{p^{*}-p}\right)\|h\|_{\infty} \frac{|\Omega|^{\frac{p^{*}+\gamma-1}{p^{*}}}}{\left(\sqrt[p]{S_{a}}\right)^{1-\gamma}}\right]^{\frac{1}{p-1+\gamma}} \\
& :=\mathrm{A}_{0} \text {. }
\end{aligned}
$$

Lemma 2.3. Assume that $\mu \in(0, \mathrm{~T})$, then $\mathcal{N}_{\mu}^{-}$is a closed set in $\mathrm{W}_{\mathrm{a}}^{1, \mathrm{p}}$-topology. 
Proof. The proof is identical to that of [21, Lemma 2], we omit it here.

Lemma 2.4. Given $v \in \mathcal{N}_{\mu}^{ \pm}$, then for every $\phi \in W_{\mathrm{a}}^{1, \mathrm{p}}(\Omega)$, there exist $\epsilon>0$ and a continuous function $\mathrm{f}(w)>$ $0, w \in W_{\mathrm{a}}^{1, \mathrm{p}}(\Omega),\|w\|<\epsilon$ such that

$$
\mathrm{f}(0)=1, \text { and } \frac{v+w \phi}{\mathrm{f}(w)} \in \mathcal{N}_{\mu}^{ \pm}, \quad \forall w \in \mathrm{W}_{\mathrm{a}}^{1, \mathrm{p}}(\Omega),\|w\|<\epsilon .
$$

Proof. The proof is identical to that of [20, Lemma 2.4], we omit it here.

\section{Solution of (1.1) for all $\mu \in(0, T)$}

Theorem 3.1. Suppose that $\mu \in(0, \mathrm{~T})$. Then the problem (1.1) has a solution $v_{0} \in \mathrm{W}_{\mathrm{a}}^{1, \mathrm{p}}(\Omega)$ satisfying $\mathrm{I}_{\mu}\left(v_{0}\right)<0$ and $\left\|v_{0}\right\|_{\mathrm{a}} \leqslant \mathrm{A}_{0}$.

Proof. For every $v \in \mathcal{N}_{\mu}$, we deduce from (2.2) that

$$
\begin{aligned}
& \mathrm{I}_{\mu}(v)=\frac{1}{\mathrm{p}} \int_{\Omega}|x|^{-\mathrm{ap}}|\nabla v|^{\mathrm{p}} \mathrm{d} x-\frac{1}{1-\gamma} \int_{\Omega} \mathrm{h}(\mathrm{x})\left(|x|^{-\mathrm{b}}|v|\right)^{1-\gamma} \mathrm{d} x-\frac{\mu}{\mathrm{p}^{*}} \int_{\Omega} \frac{|v|^{\mathrm{p}^{*}}}{|x|^{\mathrm{b} \mathrm{p}^{*}}} \mathrm{~d} x \\
& =\left(\frac{1}{p}-\frac{1}{p^{*}}\right) \int_{\Omega}|x|^{-a p}|\nabla v|^{p} d x-\left(\frac{1}{1-\gamma}-\frac{1}{p^{*}}\right) \int_{\Omega} h(x)\left(|x|^{-b}|v|\right)^{1-\gamma} d x \\
& \geqslant\left(\frac{1}{p}-\frac{1}{p^{*}}\right)\|v\|_{a}^{p}-\left(\frac{1}{1-\gamma}-\frac{1}{p^{*}}\right)\left(\frac{1}{\sqrt[p]{S_{a}}}\right)^{1-\gamma}\|h\|_{\infty}|\Omega|^{\frac{p^{*}-1+\gamma}{p^{*}}}\|v\|_{a}^{1-\gamma} .
\end{aligned}
$$

Therefore, $I_{\mu}$ is coercive and bounded below in $\mathcal{N}_{\mu}$.

By Lemma 2.3, we have that $\mathcal{N}_{\mu}^{+} \cup\{0\}$ and $\mathcal{N}_{\mu}^{-}$are two closed sets in $W_{a}^{1, p}(\Omega)$ when $\mu \in(0, T)$. In terms of Ekeland's variational principle [3], we can find a sequence $\left(v_{n}\right) \subset \mathcal{N}_{\mu}^{+} \cup\{0\}$ such that

(i) $\mathrm{I}_{\mu}\left(v_{n}\right)<\inf _{\mathcal{N}_{\mu}^{+} \cup\{0\}} \mathrm{I}_{\mu}+\frac{1}{n}$;

(ii) $\mathrm{I}_{\mu}(v) \geqslant \mathrm{I}_{\mu}\left(v_{n}\right)-\frac{1}{n}\left\|v-v_{n}\right\|, \quad \forall v \in \mathcal{N}_{\mu}^{+} \cup\{0\}$.

We may assume $v_{n} \geqslant 0$ on $\Omega \backslash\{0\}$. Since $I_{\mu}$ is bounded below in $\mathcal{N}_{\mu}$, by above property (i), we know that $\left(v_{n}\right)$ is bounded in $W_{a}^{1, p}(\Omega)$. Going if necessary to a subsequence,

$$
\begin{aligned}
& v_{n} \rightarrow v_{0} \text { weakly in } W_{a}^{1, p}(\Omega) \text { and } L^{p^{*}}(\Omega), \\
& v_{n} \rightarrow v_{0} \text { a.e. in } \Omega, \\
& v_{n} \rightarrow v_{0} \text { strongly in } L^{1-\gamma}(\Omega) .
\end{aligned}
$$

Let $v_{n}=v_{0}+w_{n}$ with $w_{n} \rightarrow 0$ weakly in $W_{a}^{1, p}(\Omega)$. For every $v \in \mathcal{N}_{\mu}^{+}$, it follows from $p>1$ that

$$
\begin{aligned}
\mathrm{I}_{\mu}(v) & =\left(\frac{1}{\mathrm{p}}-\frac{1}{\mathrm{p}^{*}}\right)\|v\|_{\mathrm{a}}^{\mathrm{p}}-\left(\frac{1}{1-\gamma}-\frac{1}{\mathrm{p}^{*}}\right) \frac{\mathrm{p}^{*}-\mathrm{p}}{\gamma+\mathrm{p}^{*}-1}\|v\|_{\mathrm{a}}^{\mathrm{p}} \\
& =\frac{\mathrm{p}^{*}-\mathrm{p}}{\mathrm{p}^{*}}\left(\frac{1}{\mathrm{p}}-\frac{1}{1-\gamma}\right)\|v\|_{\mathrm{a}}^{\mathrm{p}}<0,
\end{aligned}
$$

that is,

$$
\inf _{\mathcal{N}_{\mu}^{+} \cup\{0\}} \mathrm{I}_{\mu}=\inf _{\mathcal{N}_{\mu}^{+}} \mathrm{I}_{\mu}<0 .
$$

Moreover, $I_{\mu}\left(v_{0}\right) \leqslant \liminf _{n \rightarrow \infty} I_{\mu}\left(v_{n}\right)=\inf _{\mathcal{N}_{\mu}^{+} \cup\{0\}} I_{\mu}$. Hence, $v_{0} \not \equiv 0$ and $\left(v_{n}\right) \subset \mathcal{N}_{\mu}^{+}$. 
Claim 1. $v_{0}(x)>0$ a.e. in $\Omega$ and

$$
\int_{\Omega} h(x)\left(|x|^{-b}\right)^{1-\gamma} v_{0}^{-\gamma} \phi d x \leqslant \int_{\Omega}|x|^{-a p}\left|\nabla v_{0}\right|^{p-2} \nabla v_{0} \nabla \phi d x-\mu \int_{\Omega} \frac{v_{0}^{p^{*}-1}}{|x|^{b p^{*}}} \phi d x, \quad \forall \phi \in W_{a}^{1, p}(\Omega), \quad \phi \geqslant 0 .
$$

For $\mathrm{n}$ sufficiently large and a suitable positive constant $C_{1}$, we have

$$
\left(p^{*}-p\right)\left\|v_{n}\right\|_{a}^{p}-\left(\gamma+p^{*}-1\right) \int_{\Omega} h(x)\left(|x|^{-b} v_{n}\right)^{1-\gamma} d x \leqslant-C_{1}<0 .
$$

Let $\phi \in W_{0}^{1, p}(\Omega)$ with $\phi \geqslant 0$. By Lemma 2.4, we can find a continuous function $f_{n}(t)$ satisfying $f_{n}(0)=1$ and $\frac{1}{f_{n}(t)}\left(v_{n}+t \phi\right) \in \mathcal{N}_{\mu}^{+}$for every $v_{n}$. It follows from $v_{n} \in \mathcal{N}_{\mu}$ and $\frac{1}{f_{n}(t)}\left(v_{n}+t \phi\right) \in \mathcal{N}_{\mu}$ that

$$
\left\|v_{n}\right\|_{a}^{p}-\int_{\Omega} h(x)\left(|x|^{-b} v_{n}\right)^{1-\gamma} d x-\mu \int_{\Omega}\left(|x|^{-b} v_{n}\right)^{p^{*}} d x=0
$$

and

$$
\frac{1}{f_{n}^{p}(t)}\left\|v_{n}+t \phi\right\|_{a}^{p}-\frac{1}{f_{n}^{1-\gamma}(t)} \int_{\Omega} h(x)\left[|x|^{-b}\left(v_{n}+t \phi\right)\right]^{1-\gamma} d x-\mu \frac{1}{f_{n}^{p^{*}}(t)} \int_{\Omega}\left[|x|^{-b}\left(v_{n}+t \phi\right)\right]^{p^{*}} d x=0,
$$

so we have that

$$
\begin{aligned}
0= & \left(\frac{1}{f_{n}^{p}(t)}-1\right)\left\|v_{n}+t \phi\right\|_{a}^{p}+\left(\left\|v_{n}+t \phi\right\|_{a}^{p}-\left\|v_{n}\right\|_{a}^{p}\right) \\
& -\left(\frac{1}{f_{n}^{1-\gamma}(t)}-1\right) \int_{\Omega} h(x)\left[|x|^{-b}\left(v_{n}+t \phi\right)\right]^{1-\gamma} d x \\
& -\int_{\Omega} h(x)\left[\left(|x|^{-b}\left(v_{n}+t \phi\right)\right)^{1-\gamma}-\left(|x|^{-b} v_{n}\right)^{1-\gamma}\right] d x \\
& -\mu\left(\frac{1}{f_{n}^{p^{*}}(t)}-1\right) \int_{\Omega}\left[|x|^{-b}\left(v_{n}+t \phi\right)\right]^{p^{*}} d x-\mu \int_{\Omega}\left[\left(|x|^{-b}\left(v_{n}+t \phi\right)\right)^{p^{*}}-\left(|x|^{-b} v_{n}\right)^{p^{*}}\right] d x \\
\leqslant & \left(\frac{1}{f_{n}^{p}(t)}-1\right)\left\|v_{n}+t \phi\right\|_{a}^{p}+\left(\left\|v_{n}+t \phi\right\|_{a}^{p}-\left\|v_{n}\right\|_{a}^{p}\right) \\
& -\left(\frac{1}{f_{n}^{1-\gamma}(t)}-1\right) \int_{\Omega} h(x)\left[|x|^{-b}\left(v_{n}+t \phi\right)\right]^{1-\gamma} d x-\mu\left(\frac{1}{f_{n}^{p^{*}(t)}}-1\right) \int_{\Omega}\left[|x|^{-b}\left(v_{n}+t \phi\right)\right]^{p^{*}} d x .
\end{aligned}
$$

Dividing by $t>0$ and letting $t \rightarrow 0$, we conclude that

$$
\begin{aligned}
0 \leqslant & -p f_{n}^{\prime}(0)\left\|v_{n}\right\|_{a}^{p}+p \int_{\Omega}|x|^{-a p}\left|\nabla v_{n}\right|^{p-2} \nabla v_{n} \nabla \phi d x \\
& +(1-\gamma) f_{n}^{\prime}(0) \int_{\Omega} h(x)\left(|x|^{-b} v_{n}\right)^{1-\gamma} d x+\mu p^{*} f_{n}^{\prime}(0) \int_{\Omega}\left(|x|^{-b} v_{n}\right)^{p^{*}} d x \\
= & f_{n}^{\prime}(0)\left[\left(p^{*}-p\right)\left\|v_{n}\right\|_{a}^{p}-\left(\gamma+p^{*}-1\right) \int_{\Omega} h(x)\left(|x|^{-b} v_{n}\right)^{1-\gamma} d x\right] \\
& +p \int_{\Omega}|x|^{-a p}\left|\nabla v_{n}\right|^{p-2} \nabla v_{n} \nabla \phi d x .
\end{aligned}
$$

It follows from (3.1) and (3.2) that $f_{\mathfrak{n}}^{\prime}(0) \neq+\infty$. Now we show that $f_{\mathfrak{n}}^{\prime}(0) \neq-\infty$. If no, we assume that $f_{n}^{\prime}(0)=-\infty$. Since

$$
\begin{aligned}
\left|\frac{1}{f_{n}(t)}-1\right|\left\|v_{n}\right\|_{a}+\frac{t}{f_{n}(t)}\|\phi\|_{a} & \geqslant\left\|\left(\frac{1}{f_{n}(t)}-1\right) v_{n}+\frac{t}{f_{n}(t)} \phi\right\|_{a} \\
& =\left\|\frac{1}{f_{n}(t)}\left(v_{n}+t \phi\right)-v_{n}\right\|_{a},
\end{aligned}
$$


and $f_{n}(t)>f_{n}(0)=1$ for $n$ sufficiently large, using condition (ii) with $v=\frac{1}{f_{n}(t)}\left(v_{n}+t \phi\right) \in \mathcal{N}_{\mu}^{+}$, we obtain that

$$
\begin{aligned}
& \mid \frac{1}{f_{n}(t)}-1 \mid \frac{\left\|v_{n}\right\|_{a}}{n}+\frac{t}{f_{n}(t)} \frac{\|\phi\|_{a}}{n} \\
& \geqslant \frac{1}{n}\left\|\frac{1}{f_{n}(t)}\left(v_{n}+t \phi\right)-v_{n}\right\|_{a} \\
& \geqslant I_{\mu}\left(v_{n}\right)-I_{\mu}\left[\frac{1}{f_{n}(t)}\left(v_{n}+t \phi\right)\right] \\
&= \frac{1}{p}\left\|v_{n}\right\|_{a}^{p}-\frac{1}{1-\gamma} \int_{\Omega} h(x)\left(|x|^{-b} v_{n}\right)^{1-\gamma} d x-\frac{1}{p^{*}}\left[\left\|v_{n}\right\|_{a}^{p}-\int_{\Omega} h(x)\left(|x|^{-b} v_{n}\right)^{1-\gamma} d x\right] \\
&-\frac{1}{p} \frac{1}{f_{n}^{p}(t)}\left\|v_{n}+t \phi\right\|_{a}^{p}+\frac{1}{1-\gamma} \frac{1}{f_{n}^{1-\gamma}(t)} \int_{\Omega} h(x)\left[|x|^{-b}\left(v_{n}+t \phi\right)\right]^{1-\gamma} d x \\
&+\frac{1}{p^{*}}\left[\frac{1}{f_{n}^{p}(t)}\left\|v_{n}+t \phi\right\|_{a}^{p}-\frac{1}{f_{n}^{1-\gamma}(t)} \int_{\Omega} h(x)\left[|x|^{-b}\left(v_{n}+t \phi\right)\right]^{1-\gamma} d x\right] \\
&=\left(\frac{1}{p}-\frac{1}{p^{*}}\right)\left\|v_{n}\right\|_{a}^{p}-\left(\frac{1}{p}-\frac{1}{p^{*}}\right) \frac{1}{f_{n}^{p}(t)}\left\|v_{n}+t \phi\right\|_{a}^{p} \\
&+\left(\frac{1}{p^{*}}-\frac{1}{1-\gamma}\right) \int_{\Omega} h(x)\left(|x|^{-b} v_{n}\right)^{1-\gamma} d x \\
&-\left(\frac{1}{p^{*}}-\frac{1}{1-\gamma}\right) \frac{1}{f_{n}^{1-\gamma}(t)} \int_{\Omega} h(x)\left[|x|^{-b}\left(v_{n}+t \phi\right)\right]^{1-\gamma} d x \\
&=\left(\frac{1}{p^{*}}-\frac{1}{p}\right)\left[\left\|v_{n}+t \phi\right\|_{a}^{p}-\left\|v_{n}\right\|_{a}^{p}\right]+\left(\frac{1}{p}-\frac{1}{p^{*}}\right)\left(1-\frac{1}{f_{n}^{p}(t)}\right)\left\|v_{n}+t \phi\right\|_{a}^{p} \\
&-\left(\frac{1}{p^{*}}-\frac{1}{1-\gamma}\right) \frac{1}{f_{n}^{1-\gamma}(t)} \int_{\Omega} h(x)\left[\left(|x|^{-b}\left(v_{n}+t \phi\right)\right)^{1-\gamma}-\left(|x|^{-b} v_{n}\right)^{1-\gamma}\right] d x \\
&+\left(\frac{1}{p^{*}}-\frac{1}{1-\gamma}\right)\left(1-\frac{1}{f_{n}^{1-\gamma}(t)}\right) \int_{\Omega} h(x)\left(|x|^{-b} v_{n}\right)^{1-\gamma} d x . \\
&
\end{aligned}
$$

Dividing by $t>0$, and letting $t \rightarrow 0$, we know that

$$
\begin{aligned}
f_{n}^{\prime}(0) \frac{\left\|v_{n}\right\|_{a}}{n}+\frac{\|\phi\|_{a}}{n} \geqslant & \frac{f_{n}^{\prime}(0)}{p^{*}}\left[\left(p^{*}-p\right)\left\|v_{n}\right\|_{a}^{p}+\left(1-\gamma-p^{*}\right) \int_{\Omega} h(x)\left(|x|^{-b} v_{n}\right)^{1-\gamma} d x\right] \\
& +\left(\frac{p-p^{*}}{p^{*}}\right) \int_{\Omega}|x|^{-a p}\left|\nabla v_{n}\right|^{p-2} \nabla v_{n} \nabla \phi d x,
\end{aligned}
$$

that is

$$
\begin{aligned}
\frac{\|\phi\|_{\mathrm{a}}}{\mathrm{n}} \geqslant & \frac{f_{n}^{\prime}(0)}{\mathrm{p}^{*}}\left[\left(\mathrm{p}^{*}-\mathrm{p}\right)\left\|v_{\mathrm{n}}\right\|_{\mathrm{a}}^{\mathrm{p}}+\left(1-\gamma-\mathrm{p}^{*}\right) \int_{\Omega} \mathrm{h}(\mathrm{x})\left[|x|^{-\mathrm{b}} v_{\mathrm{n}}\right]^{1-\gamma} \mathrm{d} x-\frac{\mathrm{p}^{*}}{\mathrm{n}}\left\|v_{\mathrm{n}}\right\|_{\mathrm{a}}\right] \\
& +\int_{\Omega}|x|^{-\mathrm{ap}}\left|\nabla v_{\mathrm{n}}\right|^{\mathrm{p}-2} \nabla v_{\mathrm{n}} \nabla \phi \mathrm{d} x
\end{aligned}
$$

which contradicts with $f_{n}^{\prime}(0)=-\infty$ and

$$
\left(p^{*}-p\right)\left\|v_{n}\right\|_{a}^{p}+\left(1-\gamma-p^{*}\right) \int_{\Omega} h(x)\left[|x|^{-b} v_{n}\right]^{1-\gamma} d x-\frac{p^{*}}{n}\left\|v_{n}\right\|_{a} \leqslant-C_{2}<0 .
$$

In conclusion, $\left|f_{n}^{\prime}(0)\right|<+\infty$. It follows from (3.1), (3.2) and (3.4) that $\left|f_{n}^{\prime}(0)\right| \leqslant C_{3}$ for $n$ sufficiently large and a suitable positive constant $\mathrm{C}_{3}$. 
Now, by (3.3) and condition (ii), we get

$$
\begin{aligned}
& \frac{1}{n}\left[\left|\frac{1}{f_{n}(t)}-1\right|\left\|v_{n}\right\|_{a}+t \frac{1}{f_{n}(t)}\|\phi\|_{a}\right] \\
& \geqslant I_{\mu}\left(v_{n}\right)-I_{\mu}\left[\frac{1}{f_{n}(t)}\left(v_{n}+t \phi\right)\right] \\
&= \frac{1}{p}\left\|v_{n}\right\|_{a}^{p}-\frac{1}{1-\gamma} \int_{\Omega} h(x)\left[|x|^{-b} v_{n}\right]^{1-\gamma} d x-\frac{\mu}{p^{*}} \int_{\Omega}\left[|x|^{-b} v_{n}\right]^{p^{*}} d x-\frac{1}{p} \frac{1}{f_{n}^{p}(t)}\left\|v_{n}+t \phi\right\|_{a}^{p} \\
&+\frac{1}{(1-\gamma) f_{n}^{1-\gamma}(t)} \int_{\Omega} h(x)\left[|x|^{-b}\left|v_{n}+t \phi\right|\right]^{1-\gamma} d x+\frac{\mu}{p^{*} f_{n}^{p^{*}}(t)} \int_{\Omega}\left[|x|^{-b}\left|v_{n}+t \phi\right|\right]^{p^{*}} d x \\
&=-\frac{1}{p f_{n}^{p}(t)}\left[\left\|v_{n}+t \phi\right\|_{a}^{p}-\left\|v_{n}\right\|_{a}^{p}\right]+\left(\frac{f_{n}^{p}(t)-1}{p f_{n}^{p}(t)}\right)\left\|v_{n}\right\|_{a}^{p} \\
&+\frac{1}{(1-\gamma) f_{n}^{1-\gamma}(t)} \int_{\Omega} h(x)\left[\left(|x|^{-b}\left(v_{n}+t \phi\right)\right)^{1-\gamma}-\left(|x|^{-b} v_{n}\right)^{1-\gamma}\right] d x \\
&+\left(\frac{1-f_{n}^{1-\gamma}(t)}{(1-\gamma) f_{n}^{1-\gamma}(t)}\right) \int_{\Omega} h(x)\left[|x|^{-b} v_{n}\right]^{1-\gamma} d x \\
&+\frac{\mu}{p^{*} f_{n}^{p^{*}}(t)} \int_{\Omega}\left[\left(|x|^{-b}\left(v_{n}+t \phi\right)\right)^{p^{*}}-\left(|x|^{-b} v_{n}\right)^{p^{*}}\right] d x+\left(\frac{\mu\left(1-f_{n}^{p^{*}}(t)\right)}{p^{*} f_{n}^{p^{*}}(t)}\right) \int_{\Omega}\left(|x|^{-b} v_{n}\right)^{p^{*}} d x .
\end{aligned}
$$

Dividing by $t>0$, and passing to the limit as $t \rightarrow 0^{+}$, we get

$$
\begin{aligned}
\frac{1}{n}\left[\mid f_{n}^{\prime}(0)\left\|v_{n}\right\|_{a}+\|\phi\|_{a}\right] & \\
\geqslant & f_{n}^{\prime}(0)\left[\left\|v_{n}\right\|_{a}^{p}-\int_{\Omega} h(x)\left[|x|^{-b} v_{n}\right]^{1-\gamma} \mathrm{d} x-\mu \int_{\Omega}\left[|x|^{-b} v_{n}\right]^{p^{*}} d x\right]-\int_{\Omega}|x|^{-a p}\left|\nabla v_{n}\right|^{p-2} \nabla v_{n} \nabla \phi d x \\
& +\mu \int_{\Omega}\left(|x|^{-b}\right)^{p^{*}}\left(v_{n}\right)^{p^{*}-1} \phi d x+\liminf _{\mathrm{t} \rightarrow 0^{+}} \frac{1}{1-\gamma} \int_{\Omega} \frac{h(x)\left[\left(|x|^{-b}\left(v_{n}+t \phi\right)\right)^{1-\gamma}-\left(|x|^{-b} v_{n}\right)^{1-\gamma}\right]}{t} d x \\
= & -\int_{\Omega}|x|^{-a p}\left|\nabla v_{n}\right|^{p-2} \nabla v_{n} \nabla \phi d x+\mu \int_{\Omega}\left(|x|^{-b}\right)^{p^{*}}\left(v_{n}\right)^{p^{*}-1} \phi d x \\
& +\liminf _{t \rightarrow 0^{+}} \frac{1}{1-\gamma} \int_{\Omega} \frac{h(x)\left[\left(|x|^{-b}\left(v_{n}+t \phi\right)\right)^{1-\gamma}-\left(|x|^{-b} v_{n}\right)^{1-\gamma}\right]}{t} d x,
\end{aligned}
$$

which yields,

$$
\begin{aligned}
& \liminf _{t \rightarrow 0^{+}} \frac{1}{1-\gamma} \int_{\Omega} \frac{h(x)\left[\left(|x|^{-b}\left(v_{n}+t \phi\right)\right)^{1-\gamma}-\left(|x|^{-b} v_{n}\right)^{1-\gamma}\right]}{t} d x \\
& \leqslant \int_{\Omega}|x|^{-a p}\left|\nabla v_{n}\right|^{p-2} \nabla v_{n} \nabla \phi d x-\mu \int_{\Omega}\left(|x|^{-b}\right)^{p^{*}}\left(v_{n}\right)^{p^{*}-1} \phi d x+\frac{\left|f_{n}^{\prime}(0)\right|\left\|v_{n}\right\|_{a}+\|\phi\|_{a}}{n} .
\end{aligned}
$$

Applying Fatou's Lemma, we have

$$
\begin{aligned}
& \int_{\Omega} \liminf _{\mathrm{t} \rightarrow 0^{+}}\left[\frac{1}{1-\gamma} \frac{h(x)\left[\left(|x|^{-b}\left(v_{n}+\mathrm{t} \phi\right)\right)^{1-\gamma}-\left(|x|^{-b} v_{n}\right)^{1-\gamma}\right]}{t}\right] d x \\
& \quad \leqslant \int_{\Omega}|x|^{-a p}\left|\nabla v_{n}\right|^{p-2} \nabla v_{n} \nabla \phi d x-\mu \int_{\Omega}\left(|x|^{-b}\right)^{p^{*}}\left(v_{n}\right)^{p^{*}-1} \phi d x+\frac{\left|f_{n}^{\prime}(0)\right|\left\|v_{n}\right\|_{a}+\|\phi\|_{a}}{n} .
\end{aligned}
$$

We take $\phi=e_{1}$ as a test-function in (3.5), we know that $\nu_{n}(t)>0$ a.e. in $\Omega$, then

$$
\begin{aligned}
\int_{\Omega} h(x)\left(|x|^{-b}\right)^{1-\gamma}\left(v_{n}\right)^{-\gamma} \phi d x \leqslant & \int_{\Omega}|x|^{-a p}\left|\nabla v_{n}\right|^{p-2} \nabla v_{n} \nabla \phi d x \\
& -\mu \int_{\Omega}\left(|x|^{-b}\right)^{p^{*}}\left(v_{n}\right)^{p^{*}-1} \phi d x+\frac{\left|f_{n}^{\prime}(0)\right|\left\|v_{n}\right\|_{a}+\|\phi\|_{a}}{n},
\end{aligned}
$$


and note that $f_{n}^{\prime}(0)$ is uniformly bounded in $n$, we can deduce that $v_{0}(x)>0$ a.e. in $\Omega$, and

$$
\begin{aligned}
\int_{\Omega} h(x)\left(|x|^{-b}\right)^{1-\gamma}\left(v_{0}\right)^{-\gamma} \phi d x \leqslant & \int_{\Omega}|x|^{-a p}\left|\nabla v_{0}\right|^{p-2} \nabla v_{0} \nabla \phi d x \\
& -\mu \int_{\Omega}\left(|x|^{-b}\right)^{p^{*}}\left(v_{0}\right)^{p^{*}-1} \phi d x, \quad \forall \phi \in W_{a}^{1, p}(\Omega), \quad \phi \geqslant 0 .
\end{aligned}
$$

For $c \in \Omega$ let $\eta \in \mathrm{C}_{0}^{\infty}(\Omega)$ such that $0 \leqslant \eta(x) \leqslant 1$ in $\Omega$ and $\eta(x)=1$, for all $x \in \overline{\mathrm{B}}_{\mathrm{r}}(\mathrm{c}) \subset \Omega$ for a suitable $r>0$. Set

$$
\mathrm{U}_{\varepsilon, \mathrm{c}}(x)=\epsilon^{-\frac{\mathrm{N}-\mathrm{p}}{\mathrm{p}}} \eta(x) \mathrm{U}^{*}\left(\frac{|x-\mathrm{a}|}{\epsilon}\right) \in W_{\mathrm{a}}^{1, p}(\Omega) .
$$

It is well-known that

$$
\left\|\mathrm{U}_{\epsilon, \mathrm{c}}\right\|_{\mathrm{a}}^{\mathrm{p}}=\mathrm{B}+\mathrm{O}\left(\epsilon^{\mathrm{N}-\mathrm{p}}\right), \quad\left\|\mathrm{U}_{\epsilon, \mathrm{c}}\right\|_{\mathrm{p}^{*}}^{\mathrm{p}^{*}}=\mathrm{A}+\mathrm{O}\left(\epsilon^{\mathrm{N}}\right),
$$

and $S_{a}=\frac{B}{A^{\frac{p}{p^{*}}}}$, where $B=\int_{R^{N}}|x|^{-a p}\left|\nabla U^{*}\right| p d x, A=\int_{R^{N}}\left(x^{-b}\left|U^{*}\right|\right)^{p^{*}} d x([5,23,24])$.

Claim 2. $v_{0} \in \mathcal{N}_{\mu}$ with $\mu \in(0, T)$.

Let

$$
a_{0}=\left\|v_{0}\right\|_{a}^{p}-\int_{\Omega} h(x)\left(|x|^{-b}\left|v_{0}\right|\right)^{1-\gamma} d x-\mu \int_{\Omega}\left(|x|^{-b}\left|v_{0}\right|\right)^{p^{*}} d x
$$

Take $\phi=v_{0}$ in (3.6), we have that $a_{0} \geqslant 0$. If no, and suppose that $a_{0}>0$. There exists a unique $\tau_{0}>0$ such that $\tau_{0}^{p} B-\mu \tau_{0}^{p^{*}} A=-a_{0}$. Note that $I_{\mu}\left(v_{n}\right) \rightarrow v_{0}:=\inf _{\mathcal{N}_{\mu}^{+} \cup\{0\}} I_{\mu}=\inf _{\mathcal{N}_{\mu}^{+}} I_{\mu}$ with $v_{n} \in \mathcal{N}_{\mu}^{+}\left(\subset \mathcal{N}_{\mu}\right)$, it follows from Brezis-lieb Lemma [4] that

$$
\begin{aligned}
v_{0}+\mathrm{o}(1) & =\mathrm{I}_{\mu}\left(v_{\mathrm{n}}\right) \\
& =\left(\frac{1}{\mathrm{p}}-\frac{1}{1-\gamma}\right) \int_{\Omega} \mathrm{h}(\mathrm{x})\left(|x|^{-\mathrm{b}}\left|v_{\mathrm{n}}\right|\right)^{1-\gamma} \mathrm{d} x+\mu\left(\frac{1}{\mathrm{p}}-\frac{1}{\mathrm{p}^{*}}\right)\left\|v_{\mathrm{n}}\right\|_{\mathfrak{p}^{*}}^{\mathrm{p}^{*}} \\
& =\left(\frac{1}{\mathrm{p}}-\frac{1}{1-\gamma}\right) \int_{\Omega} \mathrm{h}(\mathrm{x})\left(|x|^{-\mathrm{b}}\left|v_{0}\right|\right)^{1-\gamma} \mathrm{d} x+\mu\left(\frac{1}{\mathrm{p}}-\frac{1}{\mathrm{p}^{*}}\right)\left\|v_{0}\right\|_{\mathfrak{p}^{*}}^{p^{*}}+\mu\left(\frac{1}{\mathrm{p}}-\frac{1}{\mathrm{p}^{*}}\right)\left\|w_{\mathrm{n}}\right\|_{\mathfrak{p}^{*}}^{p^{*}}+\mathrm{o}(1),
\end{aligned}
$$

and

$$
\begin{aligned}
0 & =\left\|v_{n}\right\|_{a}^{p}-\int_{\Omega} h(x)\left(|x|^{-b}\left|v_{n}\right|\right)^{1-\gamma} d x-\mu \int_{\Omega}\left(|x|^{-b}\left|v_{n}\right|\right)^{p^{*}} d x \\
& =a_{0}+\left\|w_{n}\right\|_{a}^{p}-\mu\left\|w_{n}\right\|_{p^{*}}^{p^{*}}+o(1) \\
& \geqslant a_{0}+S_{a}\left\|w_{n}\right\|_{p^{*}}^{p}-\mu\left\|w_{n}\right\|_{p^{*}}^{p^{*}}+o(1)
\end{aligned}
$$

which means $\lim _{n \rightarrow \infty}\left\|w_{n}\right\|_{p^{*}}$ exists and $\lim _{n \rightarrow \infty}\left\|w_{n}\right\|_{p^{*}} \geqslant \tau_{0} A^{\frac{1}{p^{*}}}$. That is to say, we have

$$
v_{0} \geqslant\left(\frac{1}{p}-\frac{1}{1-\gamma}\right) \int_{\Omega} h(x)\left(|x|^{-b}\left|v_{0}\right|\right)^{1-\gamma} d x+\mu\left(\frac{1}{p}-\frac{1}{p^{*}}\right)\left\|v_{0}\right\|_{p^{*}}^{p^{*}}+\mu\left(\frac{1}{p}-\frac{1}{p^{*}}\right) \tau_{0}^{p^{*}} A
$$

For every $v \in W_{a}^{1, p}(\Omega)$ with $a_{v}=\|v\|_{a}^{p}-\int_{\Omega} h(x)\left(|x|^{-b}|v|\right)^{1-\gamma} d x-\mu\|v\|_{p^{*}}^{p^{*}}>0$, there exists $R_{v}>0$ such that $\mathrm{a}_{v}+\mathrm{R}_{v}^{\mathrm{p}} \mathrm{B}-\mu \mathrm{R}_{v}^{\mathrm{p}^{*}} \mathrm{~A}<0$, and hence

$$
\begin{aligned}
\left\|v+\mathrm{R}_{v} \mathrm{U}_{\epsilon, \mathrm{c}}\right\|_{\mathrm{a}}^{\mathrm{p}} & -\int_{\Omega} \mathrm{h}(\mathrm{x})\left(|x|^{-\mathrm{b}}\left|v+\mathrm{R}_{v} \mathrm{U}_{\epsilon, \mathrm{c}}\right|\right)^{1-\gamma} \mathrm{d} x-\mu\left\|v+\mathrm{R}_{v} \mathrm{U}_{\epsilon, \mathrm{c}}\right\|_{\mathrm{p}^{*}}^{\mathrm{p}^{*}} \\
& =\|v\|_{\mathrm{a}}^{\mathrm{p}}+\mathrm{R}_{v}^{\mathrm{p}}\left\|\mathrm{U}_{\epsilon, \mathrm{c}}\right\|_{\mathrm{a}}^{\mathrm{p}}-\int_{\Omega} \mathrm{h}(\mathrm{x})\left(|\mathrm{x}|^{-\mathrm{b}}\left|v+\mathrm{R}_{v} \mathrm{U}_{\epsilon, \mathrm{c}}\right|\right)^{1-\gamma} \mathrm{d} x-\mu\left[\|v\|_{\mathrm{p}^{*}}^{\mathrm{p}^{*}}+\mathrm{R}_{v}^{\mathrm{p}^{*}}\left\|\mathrm{U}_{\epsilon, \mathrm{c}}\right\|_{\mathrm{p}^{*}}^{\mathrm{p}^{*}}+\mathrm{o}(1)\right] \\
& =\mathrm{a}_{v}+\mathrm{R}_{v}^{\mathrm{p}} \mathrm{B}-\mu \mathrm{R}_{v}^{\mathrm{p}^{*}} \mathrm{~A}+\mathrm{o}(1)<0,
\end{aligned}
$$

for $\epsilon>0$ small enough. Consequently, we can take $0<\mathrm{d}_{\epsilon, v}<\mathrm{R}_{v}$ to hold

$$
\left\|v+\mathrm{d}_{\epsilon, v} \mathrm{u}_{\epsilon, \mathrm{c}}\right\|_{\mathrm{a}}^{\mathrm{p}}-\int_{\Omega} \mathrm{h}(\mathrm{x})\left(|x|^{-\mathrm{b}}\left|v+\mathrm{d}_{\epsilon, v} \mathrm{u}_{\epsilon, \mathrm{c}}\right|\right)^{1-\gamma} \mathrm{d} x-\mu \int_{\Omega}\left(|x|^{-\mathrm{b}}\left|v+\mathrm{d}_{\epsilon, v} \mathrm{u}_{\varepsilon, \mathrm{c}}\right|\right)^{p^{*}} \mathrm{~d} x=0 .
$$

Thus $v+d_{\varepsilon, v} u_{\varepsilon, c} \in \mathcal{N}_{\mu}$. In addition, note that $\mathrm{a}_{v}>0$, let $\mathrm{d}_{v}>0$ be the unique positive number satisfying 
$\mathrm{d}_{v}^{\mathrm{p}} \mathrm{B}-\mu \mathrm{d}_{v}^{\mathrm{p}^{*}} \mathrm{~A}=-\mathrm{a}_{v}$. By (3.6), we get

$$
a_{v}+d_{\epsilon, v}^{p} B-\mu d_{\epsilon, v}^{p^{*}} A+o(1)=0,
$$

and thus $\mathrm{d}_{\epsilon, v} \rightarrow \mathrm{d}_{v}$ as $\varepsilon \rightarrow 0$ which leads to

$$
\left\|v+\mathrm{d}_{\epsilon, v} \mathrm{U}_{\epsilon, \mathrm{c}}\right\|_{\mathrm{a}}^{\mathrm{p}}=\|v\|_{\mathrm{a}}^{\mathrm{p}}+\mathrm{d}_{v}^{\mathrm{p}} \mathrm{B}+\mathrm{o}(1)>\mathrm{d}_{v}^{\mathrm{p}} \mathrm{B}>\left(\frac{\mathrm{B}}{\mu \mathrm{A}}\right)^{\frac{\mathrm{p}}{\mathrm{p}^{*}-\mathrm{p}}} \mathrm{B}=\left(\frac{1}{\mu}\right)^{\frac{\mathrm{p}}{\mathrm{p}^{*}-\mathrm{p}}} S_{\mathrm{a}}^{\frac{\mathrm{N}}{\mathrm{p}}},
$$

for $\epsilon>0$ sufficiently small. Since

$$
\left\|v+d_{\epsilon, \nu} \mathrm{U}_{\epsilon, c}\right\|_{\mathrm{a}}>\left(\frac{1}{\mu}\right)^{\frac{1}{\mathrm{p}^{*}-\mathrm{p}}} \sqrt[p]{\mathrm{S}_{\mathrm{a}}}{ }^{\frac{\mathrm{N}}{\mathrm{p}}}>\left(\frac{\mathrm{p}+\gamma-1}{\mu\left(\gamma+\mathrm{p}^{*}-1\right)}\right)^{\frac{1}{\mathrm{p}^{*}-\mathrm{p}}} \sqrt[p]{\mathrm{S}_{\mathrm{a}}}{ }^{\frac{\mathrm{N}}{\mathrm{p}}}=A(\mu) .
$$

By Lemma 2.2, we have $v+d_{\epsilon, \nu} U_{\epsilon, c} \in \mathcal{N}_{\mu}^{-}$. Note that $\inf _{\mathcal{N}_{\mu}^{+}} I_{\mu}=\inf _{\mathcal{N}_{\mu}} I_{\mu}$, we obtain that

$$
\begin{aligned}
v_{0} & \leqslant I_{\mu}\left(v+d_{\epsilon, \nu} U_{\epsilon, c}\right) \\
& =\left(\frac{1}{p}-\frac{1}{1-\gamma}\right) \int_{\Omega} h(x)\left(|x|^{-b}\left|v+d_{\epsilon, \nu} U_{\epsilon, c}\right|\right)^{1-\gamma} d x+\mu\left(\frac{1}{p}-\frac{1}{p^{*}}\right)\left\|v+d_{\epsilon, \nu} U_{\epsilon, c}\right\|_{p^{*}}^{p^{*}} \\
& =\left(\frac{1}{p}-\frac{1}{1-\gamma}\right) \int_{\Omega} h(x)\left(|x|^{-b}|v|\right)^{1-\gamma} d x+\mu\left(\frac{1}{p}-\frac{1}{p^{*}}\right)\|v\|_{p^{*}}^{p^{*}}+\mu\left(\frac{1}{p}-\frac{1}{p^{*}}\right) d_{\epsilon, \nu}^{p^{*}} A+o(1),
\end{aligned}
$$

i.e.,

$$
v_{0} \leqslant\left(\frac{1}{p}-\frac{1}{1-\gamma}\right) \int_{\Omega} h(x)\left(|x|^{-b}|v|\right)^{1-\gamma} d x+\mu\left(\frac{1}{p}-\frac{1}{p^{*}}\right)\|v\|_{p^{*}}^{p^{*}}+\mu\left(\frac{1}{p}-\frac{1}{p^{*}}\right) d_{v}^{p^{*}} A .
$$

Combining (3.7) and (3.9), we obtain that

$$
v_{0}=\left(\frac{1}{p}-\frac{1}{1-\gamma}\right) \int_{\Omega} h(x)\left(|x|^{-b}\left|v_{0}\right|\right)^{1-\gamma} d x+\mu\left(\frac{1}{p}-\frac{1}{p^{*}}\right) \int_{\Omega}\left(|x|^{-b}\left|v_{0}\right|\right)^{p^{*}} d x+\mu\left(\frac{1}{p}-\frac{1}{p^{*}}\right) \tau_{0}^{p^{*}} A
$$

Consequently, $v_{0}$ is a local minimizer for the following functional:

$$
\left(\frac{1}{p}-\frac{1}{1-\gamma}\right) \int_{\Omega} h(x)\left(|x|^{-b}|v|\right)^{1-\gamma} d x+\mu\left(\frac{1}{p}-\frac{1}{p^{*}}\right)\|v\|_{p^{*}}^{p^{*}}+\mu\left(\frac{1}{p}-\frac{1}{p^{*}}\right) d_{v}^{p^{*}} A .
$$

For $\mathrm{d}_{v}$, let $\psi \in \mathrm{C}_{0}^{\infty}(\Omega)$ and $\mathrm{g}(\mathrm{t}):=\mathrm{d}_{v_{0}+\mathrm{t} \psi}$, we have

$$
[g(t)]^{p} B-\mu[g(t)]^{p^{*}} A=-\left[\left\|v_{0}+t \psi\right\|_{a}^{p}-\int_{\Omega} h(x)\left(|x|^{-b}\left|v_{0}+t \psi\right|\right)^{1-\gamma} d x-\mu \int_{\Omega}\left(|x|^{-b}\left|v_{0}+t \psi\right|\right)^{p^{*}} d x\right] .
$$

Since $a_{0}>0$, we have that $g(t)$ exists and $g(0)=\tau_{0}$. Therefore,

$$
\begin{aligned}
& \frac{[g(t)]]^{p} B-\mu[g(t)]^{p^{*}} A-[g(0)]^{p} B+\mu[g(0)]^{p^{*}} A}{t} \\
& =\frac{[g(t)-g(0)]\left[g^{p-1}(t)+\cdots+g^{p-1}(0)\right] B-\mu A[g(t)-g(0)]\left[g^{p^{*}-1}(t)+\cdots+g^{p^{*}-1}(0)\right]}{t} \\
& =-\frac{1}{\mathrm{t}}\left\{\left\|v_{0}+\mathrm{t} \psi\right\|_{\mathrm{a}}^{\mathrm{p}}-\int_{\Omega} \mathrm{h}(\mathrm{x})\left(|x|^{-\mathrm{b}}\left|v_{0}+\mathrm{t} \psi\right|\right)^{1-\gamma} \mathrm{d} x-\mu \int_{\Omega}\left(|x|^{-\mathrm{b}}\left|v_{0}+\mathrm{t} \psi\right|\right)^{\mathrm{p}^{*}} \mathrm{~d} x\right. \\
& \left.-\left\|v_{0}\right\|_{\mathbf{a}}^{\mathrm{p}}+\int_{\Omega} \mathrm{h}(\mathrm{x})\left(|x|^{-\mathrm{b}}\left|v_{0}\right|\right)^{1-\gamma} \mathrm{d} x+\mu \int_{\Omega}\left(|x|^{-\mathrm{b}}\left|v_{0}\right|\right)^{\mathbf{p}^{*}} \mathrm{~d} x\right\} \\
& \stackrel{\mathrm{t} \rightarrow 0}{\longrightarrow}-\left[\mathrm{p} \int_{\Omega}|x|^{-\mathrm{ap}}\left|\nabla v_{0}\right|^{\mathrm{p}-1} \nabla \psi \mathrm{d} x-(1-\gamma) \int_{\Omega} \frac{\mathrm{h}(\mathrm{x})\left(|\mathrm{x}|^{-\mathrm{b}}\right)^{1-\gamma}}{v_{0}^{\gamma}} \psi \mathrm{d} x-\mu \mathrm{p}^{*} \int_{\Omega}\left(|x|^{-\mathrm{b}}\right)^{\mathrm{p}^{*}} v_{0}^{\mathrm{p}^{*}-1} \psi \mathrm{d} x\right] .
\end{aligned}
$$


It follows that $g^{\prime}(0)$ exists and

$$
\begin{aligned}
g^{\prime}(0)= & \frac{-1}{p \tau_{0}^{p-1} B-\mu p^{*} \tau_{0}^{p^{*}-1} \mathrm{~A}}\left[\mathrm{p} \int_{\Omega}|x|^{-a p}\left|\nabla v_{0}\right|^{p-1} \nabla \psi d x-(1-\gamma) \int_{\Omega} \frac{h(x)\left(|x|^{-b}\right)^{1-\gamma}}{v_{0}^{\gamma}} \psi d x\right. \\
& \left.-\mu p^{*} \int_{\Omega}\left(|x|^{-b}\right)^{p^{*}} v_{0}^{p^{*}-1} \psi d x\right] .
\end{aligned}
$$

Noting that (3.11) yields

$$
\begin{aligned}
& \frac{d}{d t}\left\{\left(\frac{1}{p}-\frac{1}{1-\gamma}\right) \int_{\Omega} h(x)\left(|x|^{-b}\left|v_{0}+t \psi\right|\right)^{1-\gamma} d x+\mu\left(\frac{1}{p}-\frac{1}{p^{*}}\right) \int_{\Omega}\left(|x|^{-b}\left|v_{0}+t \psi\right|\right)^{p^{*}} d x\right. \\
& \left.\quad+\mu\left(\frac{1}{p}-\frac{1}{p^{*}}\right)[g(t)]^{p^{*}} A\right\}\left.\right|_{t=0}=0,
\end{aligned}
$$

we have

$$
\begin{aligned}
\left(\frac{1}{p}\right. & \left.-\frac{1}{1-\gamma}\right)(1-\gamma) \int_{\Omega} \frac{h(x)\left(|x|^{-b}\right)^{1-\gamma}}{v_{0}^{\gamma}} \psi d x \\
& +\mu\left(\frac{1}{p}-\frac{1}{p^{*}}\right) p^{*} \int_{\Omega}\left(|x|^{-b}\right)^{p^{*}} v_{0}^{p^{*}-1} \psi d x+\mu\left(\frac{1}{p}-\frac{1}{p^{*}}\right) p^{*} \tau_{0}^{p^{*}-1} A \\
& \times\left\{\frac { - 1 } { p \tau _ { 0 } ^ { p ^ { * } - 1 } B - \mu p ^ { * } \tau _ { 0 } ^ { p ^ { * } - 1 } A } \left[p \int_{\Omega}|x|^{-a p}\left|\nabla v_{0}\right|^{p-1} \nabla \psi d x-(1-\gamma) \int_{\Omega} \frac{h(x)\left(|x|^{-b}\right)^{1-\gamma}}{v_{0}^{\gamma}} \psi d x\right.\right. \\
& \left.\left.-\mu p^{*} \int_{\Omega}\left(|x|^{-b}\right)^{p^{*}} v_{0}^{p^{*}-1} \psi d x\right]\right\}=0
\end{aligned}
$$

for all $\psi \in C_{0}^{\infty}(\Omega)$. Denote $d_{\epsilon, v_{0}}=\tau_{0}+\delta_{\epsilon}$, since $d_{\epsilon, \nu} \longrightarrow d_{\nu}$, as $\epsilon \longrightarrow 0$, we have $\delta_{\epsilon} \rightarrow 0$. All the above estimates are substituted in (3.8), we have

$$
\begin{aligned}
& 0=\left\|v_{0}+\mathrm{d}_{\epsilon, v_{0}} \mathrm{u}_{\epsilon, \mathrm{c}}\right\|_{\mathbf{a}}^{\mathrm{p}}-\int_{\Omega} \mathrm{h}(\mathrm{x})\left(|x|^{-\mathrm{b}}\left|v_{0}+\mathrm{d}_{\epsilon, v_{0}} \mathrm{u}_{\epsilon, \mathrm{c}}\right|\right)^{1-\gamma} \mathrm{d} x-\mu \int_{\Omega}\left(|x|^{-\mathrm{b}}\left|v_{0}+\mathrm{d}_{\epsilon, v_{0}} \mathrm{u}_{\epsilon, \mathrm{c}}\right|\right)^{\mathrm{p}^{*}} \mathrm{~d} \mathrm{x}
\end{aligned}
$$

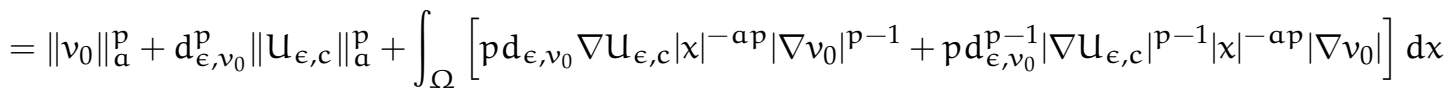

$$
\begin{aligned}
& -\int_{\Omega} h(x)\left(|x|^{-b} v_{0}\right)^{1-\gamma} d x-(1-\gamma) \tau_{0} \int_{\Omega} h(x)\left(|x|^{-b}\right)^{1-\gamma} v_{0}^{-\gamma} u_{\epsilon, c} d x \\
& -\mu\left[\int_{\Omega}\left(|x|^{-b}\right)^{p^{*}}\left(v_{0}^{p^{*}}+p^{*} c_{\epsilon, v_{0}} \mathrm{u}_{\epsilon, c} v_{0}^{p^{*}-1}+\cdots+p^{*}\left(c_{\epsilon, v_{0}} \mathrm{u}_{\epsilon, \mathrm{c}}\right)^{p^{*}-1} v_{0}+\left(\mathrm{c}_{\epsilon, v_{0}} \mathrm{u}_{\epsilon, \mathrm{c}}\right)^{p^{*}}\right) \mathrm{dx}\right]+\mathrm{o}\left(\epsilon^{\frac{\mathrm{N}-\mathrm{p}}{\mathrm{p}}}\right) \\
& =-\left(\tau_{0}^{p} B-\mu \tau_{0}^{p^{*}} A\right)+c_{\epsilon, v_{0}}^{p} B-\mu c_{\epsilon, v_{0}}^{p^{*}} A \\
& +\int_{\Omega}|x|^{-a p}\left[\mathrm{pc}_{\epsilon, v_{0}} \nabla \mathrm{U}_{\epsilon, \mathrm{c}}\left|\nabla v_{0}\right|^{\mathrm{p}-1}+\mathrm{pc}_{\epsilon, v_{0}}^{\mathrm{p}-1}\left|\nabla \mathrm{U}_{\epsilon, \mathrm{c}}\right|^{\mathrm{p}-1}\left|\nabla v_{0}\right|\right] \mathrm{d} x \\
& -(1-\gamma) \tau_{0} \int_{\Omega} h(x)\left(|x|^{-b}\right)^{1-\gamma}\left(v_{0}\right)^{-\gamma} U_{\epsilon, c} d x \\
& -\mu \int_{\Omega}\left(|x|^{-b}\right)^{p^{*}}\left(p^{*} d_{\epsilon, v_{0}} u_{\epsilon, c} v_{0}^{p^{*}-1}+p^{*} d_{\epsilon, v_{0}}^{p^{*}-1} u_{\epsilon, c}^{p^{*}-1} v_{0}\right) d x+o\left(\epsilon^{\frac{N-p}{p}}\right),
\end{aligned}
$$

which yields

$$
\begin{aligned}
{\left[p \tau_{0}^{p-1} B-\mu p^{*} \tau_{0}^{p^{*}-1} A+o(1)\right]\left(-\delta_{\epsilon}\right) } & \\
= & \int_{\Omega}|x|^{-a p}\left[p \tau_{0} \nabla u_{\epsilon, c}\left|\nabla v_{0}\right|^{p-1}+p \tau_{0}^{p-1}\left|\nabla U_{\epsilon, c}\right|^{p-1}\left|\nabla v_{0}\right|\right] d x \\
& -(1-\gamma) \tau_{0} \int_{\Omega} h(x)\left(|x|^{-b}\right)^{1-\gamma} v_{0}^{-\gamma} U_{\epsilon, c} d x \\
& -\mu \int_{\Omega}\left(|x|^{-b}\right)^{p^{*}}\left(p^{*} \tau_{0} U_{\epsilon, c} v_{0}^{p^{*}-1}+p^{*} \tau_{0}^{p^{*}-1} u_{\epsilon, c}^{p^{*}-1} v_{0}\right) d x+o\left(\epsilon^{\frac{N-p}{p}}\right) .
\end{aligned}
$$


Moreover, by (3.12), we obtain

$$
\begin{aligned}
-\delta_{\epsilon}= & \frac{\tau_{0}}{p \tau_{0}^{p-1} B-\mu p^{*} \tau_{0}^{p^{*}-1} A}\left[p \int_{\Omega}|x|^{-a p}\left|\nabla v_{0}\right|^{p-1} \nabla u_{\epsilon, c} d x\right] \\
& -\frac{\tau_{0}}{p \tau_{0}^{p-1} B-\mu p^{*} \tau_{0}^{p^{*}-1} A}(1-\gamma) \int_{\Omega} \frac{h(x)\left(|x|^{-b}\right)^{1-\gamma}}{v_{0}^{\gamma}} U_{\epsilon, c} d x \\
& -\frac{\tau_{0}}{p \tau_{0}^{p-1} B-\tau_{0}^{p^{*}-1} A} \mu p^{*} \int_{\Omega}\left(|x|^{-b}\right)^{p^{*}} v_{0}^{p^{*}-1} u_{\epsilon, c} d x \\
& +\frac{\tau_{0}^{p-1}}{p \tau_{0}^{p-1} B-\mu p^{*} \tau_{0}^{p^{*}-1} A}\left[p \int_{\Omega}|x|^{-a p}\left|\nabla U_{\epsilon, c}\right|^{p-1}\left|\nabla v_{0}\right| d x\right] \\
& -\frac{\tau_{0}^{p^{*}-1}}{p \tau_{0}^{p-1} B-\mu p^{*} \tau_{0}^{p^{*}-1} A} \mu p^{*} \int_{\Omega}\left(|x|^{-b}\right)^{p^{*}} u_{\epsilon, c}^{p^{*}-1} v_{0} d x+o\left(\epsilon \frac{N-p}{p}\right) \\
= & \tau_{0} \frac{\left(\frac{1}{p}-\frac{1}{1-\gamma}\right)(1-\gamma) \int_{\Omega} \frac{h(x)\left(|x|^{-b}\right)^{1-\gamma}}{v_{0}^{\gamma}} U_{\epsilon, c} d x+\mu\left(\frac{1}{p}-\frac{1}{p^{*}}\right) p^{*} \int_{\Omega}\left(|x|^{-b}\right)^{p^{*}} v_{0}^{p^{*}-1} U_{\epsilon, c} d x}{\mu\left(\frac{1}{p}-\frac{1}{p^{*}}\right) p^{*} \tau_{0}^{p^{*}-1} A} \\
& +\frac{\tau_{0}^{p-1}}{p \tau_{0}^{p-1} B-\mu p^{*} \tau_{0}^{p^{*}-1} A}\left[p \int_{\Omega}^{\left.|x|^{-a p}\left|\nabla U_{\epsilon, c}\right|^{p-1}\left|\nabla v_{0}\right| d x\right]}\right. \\
& -\frac{\tau_{0}^{p-1}}{p \tau_{0}^{p-1} B-\mu p^{*} \tau_{0}^{p^{*}-1} A} \mu p^{*} \int_{\Omega}\left(|x|^{-b}\right)^{p^{*}} U_{\epsilon, c}^{p^{*}-1} v_{0} d x+o\left(\epsilon \frac{N-p}{p}\right) .
\end{aligned}
$$

Since $a_{0}>0$, we have

$$
p \tau_{0}^{p-1} B-\mu p^{*} \tau_{0}^{p^{*}-1} A=\frac{p}{\tau_{0}}\left(\tau_{0}^{p} B-\mu \frac{p^{*}}{p} \tau_{0}^{p^{*}} A\right)<\frac{p}{\tau_{0}}\left(\tau_{0}^{p} B-\mu \tau_{0}^{p^{*}} A\right)=-\frac{p}{\tau_{0}} a_{0}<0 .
$$

And then, it follows from $v_{0}+\mathrm{d}_{\epsilon, v_{0}} \mathrm{u}_{\epsilon, \mathrm{c}} \in \mathcal{N}_{\mu}$, (3.10) and (3.13) that

$$
\begin{aligned}
& \mathrm{I}_{\mu}\left(v_{0}+\mathrm{d}_{\epsilon, v_{0}} \mathrm{U}_{\epsilon, \mathrm{c}}\right)=\left(\frac{1}{\mathrm{p}}-\frac{1}{1-\gamma}\right) \int_{\Omega} \mathrm{h}(\mathrm{x})\left(|x|^{-\mathrm{b}}\left|v_{0}+\mathrm{d}_{\epsilon, v_{0}} \mathrm{u}_{\epsilon, \mathrm{c}}\right|\right)^{1-\gamma} \mathrm{dx} \\
& +\mu\left(\frac{1}{p}-\frac{1}{p^{*}}\right) \int_{\Omega}\left(|x|^{-b}\left|v_{0}+d_{\epsilon, v_{0}} u_{\epsilon, c}\right|\right)^{p^{*}} d x \\
& =\left(\frac{1}{p}-\frac{1}{1-\gamma}\right) \int_{\Omega} h(x)\left(|x|^{-b} v_{0}\right)^{1-\gamma} d x \\
& +\left(\frac{1}{p}-\frac{1}{1-\gamma}\right)\left[(1-\gamma) \tau_{0} \int_{\Omega} h(x)\left(|x|^{-b}\right)^{1-\gamma} v_{0}^{-\gamma} U_{\epsilon, c} d x\right] \\
& +\mu\left(\frac{1}{p}-\frac{1}{p^{*}}\right) \int_{\Omega}\left(|x|^{-b}\left|v_{0}\right|\right)^{p^{*}} d x \\
& +\mu\left(\frac{1}{p}-\frac{1}{p^{*}}\right) d_{\epsilon, v_{0}}^{p^{*}} A+\mu\left(\frac{1}{p}-\frac{1}{p^{*}}\right) p^{*} \int_{\Omega}\left(|x|^{-b}\right)^{p^{*}} v_{0}^{p^{*}-1} d_{\epsilon, v_{0}} u_{\epsilon, c} d x \\
& +\mu\left(\frac{1}{p}-\frac{1}{p^{*}}\right) p^{*} d_{\epsilon, v_{0}}^{p^{*}-1} \int_{\Omega} v_{0} U_{\epsilon, c}^{p^{*}-1} d x+o\left(\epsilon^{\frac{N-p}{p}}\right) \\
& =\left(\frac{1}{p}-\frac{1}{1-\gamma}\right) \int_{\Omega} h(x)\left(|x|^{-b} v_{0}\right)^{1-\gamma} d x \\
& +\left(\frac{1}{p}-\frac{1}{1-\gamma}\right)\left[(1-\gamma) \tau_{0} \int_{\Omega} h(x)\left(|x|^{-b}\right)^{1-\gamma} v_{0}^{-\gamma} U_{\epsilon, c} d x\right] \\
& +\mu\left(\frac{1}{p}-\frac{1}{p^{*}}\right) \int_{\Omega}\left(|x|^{-b}\left|v_{0}\right|\right)^{p^{*}} d x+\mu\left(\frac{1}{p}-\frac{1}{p^{*}}\right) \tau_{0}^{p^{*}} A+\mu\left(\frac{1}{p}-\frac{1}{p^{*}}\right) p^{*} \tau_{0}^{p^{*}-1} \delta_{\epsilon} A
\end{aligned}
$$




$$
\begin{aligned}
& +\mu\left(\frac{1}{p}-\frac{1}{p^{*}}\right) p^{*} \tau_{0} \int_{\Omega}\left(|x|^{-b}\right)^{p^{*}} v_{0}^{p^{*}-1} u_{\epsilon, c} d x \\
& +\mu\left(\frac{1}{p}-\frac{1}{p^{*}}\right) p^{*} \tau_{0}^{p^{*}-1} \int_{\Omega}\left(|x|^{-b}\right)^{p^{*}} v_{0} u_{\epsilon, c}^{p^{*}-1} d x \\
& +\mu\left(\frac{1}{p}-\frac{1}{p^{*}}\right) p^{*}\left(p^{*}-1\right) \tau_{0}^{p^{*}-2} \delta_{\epsilon} \int_{\Omega}\left(|x|^{-b}\right)^{p^{*}} v_{0} u_{\epsilon, c}^{p^{*}-1} d x+o\left(\epsilon^{\frac{N-p}{p}}\right) \\
& =v_{0}+\left(\frac{1}{p}-\frac{1}{1-\gamma}\right)\left[(1-\gamma) \tau_{0} \int_{\Omega} h(x)\left(|x|^{-b}\right)^{1-\gamma} v_{0}^{-\gamma} \mathrm{U}_{\epsilon, \mathrm{c}} \mathrm{dx}\right] \\
& +\mu\left(\frac{1}{p}-\frac{1}{p^{*}}\right) p^{*} \tau_{0}^{p^{*}-1} \delta_{\epsilon} A+\mu\left(\frac{1}{p}-\frac{1}{p^{*}}\right) p^{*} \tau_{0} \int_{\Omega}\left(|x|^{-b}\right)^{p^{*}} v_{0}^{p^{*}-1} u_{\epsilon, c} d x \\
& +\mu\left(\frac{1}{p}-\frac{1}{p^{*}}\right) p^{*} \tau_{0}^{p^{*}-1} \int_{\Omega}\left(|x|^{-b}\right)^{p^{*}} v_{0} u_{\epsilon, c}^{p^{*}-1} d x \\
& +\mu\left(\frac{1}{p}-\frac{1}{p^{*}}\right) p^{*}\left(p^{*}-1\right) \tau_{0}^{p^{*}-2} \delta_{\epsilon} \int_{\Omega}\left(|x|^{-b}\right)^{p^{*}} v_{0} u_{\epsilon, c}^{p^{*}-1} d x+o\left(\epsilon^{\frac{N-p}{p}}\right) \\
& =v_{0}+\mu\left(\frac{1}{p}-\frac{1}{p^{*}}\right) p^{*} \tau_{0}^{p^{*}-1} \frac{p \tau_{0}^{p^{*}-1} B}{p \tau_{0}^{p^{*}-1} B-\mu p^{*} \tau_{0}^{p^{*}-1} A} \int_{\Omega}\left(|x|^{-b}\right)^{p^{*}} \nu_{0} u_{\epsilon, c}^{p^{*}-1} d x \\
& -\left(p^{*}-1\right)\left[\left(\frac{1}{p}-\frac{1}{1-\gamma}\right)(1-\gamma) \int_{\Omega} \frac{h(x)\left(|x|^{-b}\right)^{1-\gamma}}{v_{0}^{\gamma}} u_{\epsilon, c} d x\right. \\
& \left.+\mu\left(\frac{1}{p}-\frac{1}{p^{*}}\right) p^{*} \int_{\Omega}\left(|x|^{-b}\right)^{p^{*}} v_{0}^{p^{*}-1} u_{\epsilon, c} d x\right] \int_{\Omega}\left(|x|^{-b}\right)^{p^{*}} v_{0} u_{\epsilon, c}^{p^{*}-1} d x \\
& +\frac{\tau_{0}^{p^{*}-1}}{p \tau_{0}^{p^{*}-1} B-\mu p^{*} \tau_{0}^{p^{*}-1} A} \mu p^{*}\left(\int_{\Omega}\left(|x|^{-b}\right)^{p^{*}} u_{\epsilon, c}^{p^{*}-1} v_{0} d x\right) \\
& \times \mu\left(\frac{1}{p}-\frac{1}{p^{*}}\right) p^{*}\left(p^{*}-1\right) \tau_{0}^{p^{*}-2} \int_{\Omega}\left(|x|^{-b}\right)^{p^{*}} v_{0} u_{\epsilon, c}^{p^{*}-1} d x+o\left(\epsilon^{\frac{N-p}{p}}\right)<v_{0},
\end{aligned}
$$

which is a contradiction. This concludes the proof of Claim 2.

Claim 3. $v_{0}$ is a solution of (1.1).

For $\psi \in W_{a}^{1, p}(\Omega)$ and $\epsilon>0$, we define

$$
\Psi:=\left(v_{0}+\epsilon \psi\right)^{+} \in W_{0}^{1, p}(\Omega) .
$$

Equation (3.14) is substituted in (3.6), in view of Claim 2, we find that

$$
\begin{aligned}
0= & \int_{\Omega}\left(|x|^{-a p}\left|\nabla v_{0}\right|^{p-2} \nabla v_{0} \nabla \Psi-\frac{h(x)\left(|x|^{-b}\right)^{1-\gamma}}{v_{0}^{\gamma}} \Psi-\mu\left(|x|^{-b} v_{0}\right)^{p^{*}-1} \Psi\right) d x \\
= & \int_{\left[v_{0}+\epsilon \psi>0\right]}\left[|x|^{-a p}\left|\nabla v_{0}\right|^{p-2} \nabla v_{0} \nabla\left(v_{0}+\epsilon \psi\right)\right. \\
& \left.-\frac{h(x)\left(|x|^{-b}\right)^{1-\gamma}}{v_{0}^{\gamma}}\left(v_{0}+\epsilon \psi\right)-\mu\left(|x|^{-b}\right)^{p^{*}} v_{0}^{p^{*}-1}\left(v_{0}+\epsilon \psi\right)\right] d x \\
= & \int_{\Omega}\left[|x|^{-a p}\left|\nabla v_{0}\right|^{p-2} \nabla v_{0} \nabla\left(v_{0}+\epsilon \psi\right)-\frac{h(x)\left(|x|^{-b}\right)^{1-\gamma}}{v_{0}^{\gamma}}\left(v_{0}+\epsilon \psi\right)-\mu\left(|x|^{-b}\right)^{p^{*}} v_{0}^{p^{*}-1}\left(v_{0}+\epsilon \psi\right)\right] d x \\
& -\int_{\left[v_{0}+\epsilon \psi \leqslant 0\right]}\left[|x|^{-a p}\left|\nabla v_{0}\right|^{p-2} \nabla v_{0} \nabla\left(v_{0}+\epsilon \psi\right)\right. \\
& \left.-\frac{h(x)\left(|x|^{-b}\right)^{1-\gamma}}{v_{0}^{\gamma}}\left(v_{0}+\epsilon \psi\right)-\mu\left(|x|^{-b}\right)^{p^{*}} v_{0}^{p^{*}-1}\left(v_{0}+\epsilon \psi\right)\right] \mathrm{d} x \\
= & \left\|v_{0}\right\|_{a}^{p}-\int_{\Omega} h(x)\left(|x|^{-b}\right)^{1-\gamma} v_{0}^{1-\gamma} d x-\mu \int_{\Omega}\left(|x|^{-b} v_{0}\right)^{p^{*}} d x
\end{aligned}
$$




$$
\begin{aligned}
& +\epsilon\left(\int_{\Omega}|x|^{-a p}\left|\nabla v_{0}\right|^{p-2} \nabla v_{0} \nabla \psi-\frac{h(x)\left(|x|^{-b}\right)^{1-\gamma}}{v_{0}^{\gamma}} \psi-\mu\left(|x|^{-b}\right)^{p^{*}} v_{0}^{p^{*}-1} \psi\right) d x \\
& -\int_{\left[v_{0}+\epsilon \psi \leqslant 0\right]}\left(|x|^{-a p}\left|\nabla v_{0}\right|^{p-2} \nabla v_{0} \nabla\left(v_{0}+\epsilon \psi\right)\right. \\
& \left.-\frac{h(x)\left(|x|^{-b}\right)^{1-\gamma}}{v_{0}^{\gamma}}\left(v_{0}+\epsilon \psi\right)-\mu\left(|x|^{-b}\right)^{p^{*}} v_{0}^{p^{*}-1}\left(v_{0}+\epsilon \psi\right)\right) d x \\
& \leqslant \epsilon \int_{\Omega}\left(|x|^{-a p}\left|\nabla v_{0}\right|^{p-2} \nabla v_{0} \nabla \psi-\frac{h(x)\left(|x|^{-b}\right)^{1-\gamma}}{v_{0}^{\gamma}} \psi-\mu\left(|x|^{-b}\right)^{p^{*}} v_{0}^{p^{*}-1} \psi\right) d x \\
& -\epsilon \int_{\left[v_{0}+\epsilon \psi \leqslant 0\right]}\left(|x|^{-a p}\left|\nabla v_{0}\right|^{p-2} \nabla v_{0} \nabla \psi\right) d x .
\end{aligned}
$$

Note that the measurement of $\left\{v_{0}+\epsilon \psi \leqslant 0\right\} \longrightarrow 0$ as $\epsilon \rightarrow 0$. Dividing by $\epsilon$ and passing to the limit as $\epsilon \longrightarrow 0$, and hence

$$
\int_{\Omega}\left(|x|^{-a p}\left|\nabla v_{0}\right|^{p-2} \nabla v_{0} \nabla \psi-\frac{h(x)\left(|x|^{-b}\right)^{1-\gamma}}{v_{0}^{\gamma}} \psi-\mu\left(|x|^{-b}\right)^{p^{*}} v_{0}^{p^{*}-1} \psi\right) d x \geqslant 0 .
$$

Therefore, $v_{0}$ is a solution of problem (1.1). Note that $v_{n} \rightarrow v_{0}$ weakly in $W_{a}^{1, p}(\Omega)$, we know that $\left\|v_{0}\right\| \leqslant$ $\liminf \left\|v_{n}\right\|_{a} \leqslant A_{0}$. By Claim 2 and Lemma 2.2, we see that $v_{0} \in \mathcal{N}_{\mu}^{+}$. Thus, it follows from $\mathrm{I}_{\mu}\left(v_{n}\right) \longrightarrow$ $\inf _{\mathcal{N}_{+}^{+}}^{\infty}$ that

$$
\inf _{\mathcal{N}_{\mu}^{+}} I_{\mu} \geqslant\left(\frac{1}{p}-\frac{1}{p^{*}}\right)\left\|v_{0}\right\|_{a}^{p}-\left(\frac{1}{1-\gamma}-\frac{1}{p^{*}}\right) \int_{\Omega} h(x)\left(|x|^{-b} v_{0}\right)^{1-\gamma} d x=I_{\mu}\left(v_{0}\right)
$$

Consequently, $\mathrm{I}_{\mu}\left(v_{0}\right)=\inf _{\mathcal{N}_{\mu}^{+}} \mathrm{I}_{\mu}$.

Theorem 3.2. Assume that $\mu \in(0, \mathrm{~T})$. Then the problem (1.1) has a solution $\mathrm{V}_{0} \in \mathrm{W}_{\mathrm{a}}^{1, \mathrm{p}}(\Omega)$ satisfying

$$
\left\|V_{0}\right\|_{a} \geqslant A(\mu)>A_{0}
$$

Proof. The proof of Theorem 3.2 is similar to that of Theorem 3.1, we omit it here.

\section{Acknowledgment}

This project is supported by the Natural Science Foundation of Shanxi Province (2016011003) and Science Foundation of North University of China (110246).

\section{References}

[1] B. Abdellaoui, V. Felli, I. Peral, Existence and nonexistence for quasilinear equations involving the p-Laplacian, Boll. Unione Mat. Ital. Sez. B Artic. Ric. Mat., 9 (2006), 445-484. 1

[2] C. O. Alves, A. El Hamidi, Nehari manifold and existence of positive solutions to a class of quasilinear problems, Nonlinear Anal., 60 (2005), 611-624. 1

[3] J.-P. Aubin, I. Ekeland, Applied Nonlinear Analysis, John Wiley \& Sons, Inc., New York, (1984). 3

[4] H. Brézis, E. Lieb, A relation between pointwise convergence of functional and convergence of functionals, Proc. Amer. Math. Soc., 88 (1983), 486-490. 3

[5] F. Catrina, Z.-Q. Wang, On the Caffarelli-Kohn-Nirenberg inequalities: sharp constants, existence (and nonexistence), and symmetry of extremal functions, Comm. Pure Appl. Math., 54 (2001), 229-258. 3

[6] Y.-P. Chen, J.-Q. Chen, Existence of multiple positive weak solutions and estimates for extremal values to a class of elliptic problems with Hardy term and singular nonlinearity, J. Math. Anal. Appl., 429 (2015), 873-900. 1

[7] Y.-P. Chen, J.-Q. Chen, Multiple positive solutions for a semilinear equation with critical exponent and prescribed singularity, Nonlinear Anal., 130 (2016), 121-137. 1 
[8] J.-Q. Chen, E. M. Rocha, Positive solutions for elliptic problems with critical nonlinearity and combined singularity, Math. Bohem., 135 (2010), 413-422. 1

[9] S.-Q. Cong, Y.-Z. Han, Compatibility conditions for the existence of weak solutions to a singular elliptic equation, Bound. Value Probl., 2015 (2015), 11 pages.

[10] F. J. S. A. Corrêa, A. S. S. Corrêa, G. M. Figueiredo, Positive solution for a class of pq-singular elliptic equation, Nonlinear Anal. Real World Appl., 16 (2014), 163-169. 1

[11] Z.-Y. Deng, Y.-S. Huang, On G-symmetric solutions of the quasilinear elliptic equations with singular weights and critical exponents, Scientia Sinica Mathematica., 42 (2012), 1053-1066. 1

[12] Z.-Y. Deng, Y.-S. Huang, On positive G-symmetric solutions of a weighted quasilinear elliptic equation with critical HardySobolev exponent, Acta Math. Sci. Ser. B Engl. Ed., 34 (2014), 1619-1633. 1

[13] N. Ghoussoub, C. Yuan, Multiple solutions for quasi-linear PDEs involving the critical Sobolev and Hardy exponents, Trans. Amer. Math. Soc., 352 (2000), 5703-5743. 1

[14] J. Giacomoni, I. Schindler, P. Takáč, Sobolev versus Hölder local minimizers and existence of multiple solutions for a singular quasilinear equation, Ann. Sc. Norm. Super. Pisa, Cl. Sci., 6 (2007), 117-158. 1

[15] P. Han, Quasilinear elliptic problems with critical exponents and Hardy terms, Nonlinear Anal., 61 (2005), $735-758$.

[16] Y. Jalilian, On the exitence and multiplicity of solutions for a class of singular elliptic problems, Comput. Math. Appl., 68 (2014), 664-680.

[17] D.-S. Kang, On the quasilinear elliptic problems with critical Sobolev-Hardy exponents and Hardy terms, Nonlinear Anal., 68 (2008), 1973-1985. 1

[18] D.-S. Kang, Positve solutions to the weighted critical quasilinear problems, Appl. Math. Compt., 213 (2009), 432-439. 1, 1

[19] N. H. Loc, K. Schmitt, Boundary value problems for singular elliptic equations, Rocky Mt. J. Math., 41 (2011), 555-572. 1

[20] Y.-b. Sang, An exact estimate result for a semilinear equation with critical exponent and prescribed singularity, J. Math. Anal. Appl., 447 (2017), 128-153. 2

[21] Y.-J. Sun, S.-J. Li, A nonlinear elliptic equation with critical exponent: estimates for extremal values, Nonlinear Anal., 69 (2008), 1856-1869. 1, 2

[22] Y.-J. Sun, S.-P. Wu, An exact estimate result for a class of singular equations with critical exponents, J. Funct. Anal., 260 (2011), 1257-1284. 1

[23] G. Tarantello, On nonhomogeneous elliptic equations involving critical Sobolev exponent, Ann. Inst. H. Poincaré Anal. Non Linéaire, 9 (1992), 281-304. 3

[24] S. Terracini, On positive entire solutions to a class of equations with singular coefficient and critical exponent, Adv. Differential Equations, 1 (1996), 241-264. 3

[25] L. Wang, Q.-L. Wei, D.-S. Kang, Multiple positive solutions for p-Laplace elliptic equations involving concave-convex nonlinearities and a Hardy-type term, Nonliear Anal., 74 (2011), 626-638. 1

[26] B. J. Xuan, The eightvalue problem for a singular quasilinear elliptic equation, Electron. J. Differ. Equat., 16 (2004), 1-11. 1,1 